\title{
Existential Rigidity and Many Modalities in Order-Sorted Logic
}

\author{
Ken Kaneiwa \\ Department of Electrical Engineering and Computer Science, Iwate University \\ 4-3-5 Ueda, Morioka, Iwate 020-8551, Japan \\ kaneiwa@cis.iwate-u.ac.jp
}

\begin{abstract}
Order-sorted logic is a useful tool for knowledge representation and reasoning because it enables representation of sorted terms and formulas along with partially ordered sorts (called sort-hierarchy). However, this logic cannot represent more complex sorted expressions when they are true in any possible world (as rigid) or some possible worlds (as modality) such as time, space, belief, or situation. In this study, we extend order-sorted logic by introducing existential rigidity and many modalities. In the extended logic, sorted modal formulas are interpreted over the Cartesian product of sets of possible worlds. We present a new labeled tableau calculus to check the (un)satisfiability and validity of sorted modal formulas.
\end{abstract}

\section{Introduction}

Knowledge-based systems support intelligent decisions by using the technologies of knowledge, learning, and reasoning. Such systems use knowledge representation languages for storage and reasoning engines for inferring valuable knowledge. Processing of knowledge is required to deal with conceptual knowledge (e.g., ontology) and temporal and situational dependencies of knowledge (e.g., real world data). As a tool of artificial intelligence, logical languages and deductions provide us with theoretical foundations for knowledge representation and reasoning.

Order-sorted logic $[20,9,17,26,22]$ has been known as first-order predicate logic that extends to include many sorts and their hierarchy (called sort hierarchy). In knowledge representation and reasoning, automated deduction for order-sorted logic, which offers advantages in this area, has been thoroughly studied $[6,27,28]$. These advantages include: (i) reduced search space through restriction on domains and ranges of functions, predicates, and variables [23, 24, 25], (ii) reasoning on taxonomic knowledge by means of partially

\footnotetext{
${ }^{1}$ This paper contains some results of the conference paper [13].
} 
ordered sorts $[3,14,15]$, and (iii) detection of sort errors in well-sorted formulas [18]. Similar to type-checked programs, sorted formulas ensure the reliability of knowledge representation and reasoning.

On the other hand, a number of the extensions of order-sorted logic have been proposed for logical reasoning regarding time, knowledge, and actions. Galton [7] proposed reified temporal logic that can be used to describe events and actions in sorted formulas. Based on many-sorted logic, Cruz and Crossley [4] introduced tiered logic for agents in different locations. Moreover, Shapanskykh and Treur [21] made use of the reified temporal predicate language to specify cognitive processes and behaviors of agents. These extensions have been made more sophisticated for the purposes of event description and reasoning for events, time, agents, and sorts.

To the best of our knowledge, ordinal order-sorted logic (first-order predicate logic with sort-hierarchy) and its extensions have not addressed the rigidity and multiple modalities of sorted expressions in knowledge representation and reasoning. First, rigidity and anti-rigidity of sorts are important for representing the essential property of static and dynamic conceptual knowledge not dealt with by the existing temporal logic. Therefore there is a need to consider that rigid sortal properties hold in any possible world but anti-rigid sortal properties do not hold in certain possible worlds. Second, anti-rigid sorts cause a complex combination among multiple modalities in $n$-dimensional possible worlds. This is because the truths of anti-rigid sortal properties depend on a specific world, such as time, space, belief, or situation. In the extensions of order-sorted logic, events, time, agents, and sorts are effectively described; yet the semantics and reasoning for many-dimensional modalities have not been established in the temporal logic. For example, the modal formula $\square_{\text {Agt1 } 1} \diamond_{\text {Tim }} \diamond_{\text {Sit }} F_{1}$ indicates that an agent knows the fact $F_{1}$ in a situation at a time and the modal formula $\diamond_{\text {Sit }} \square_{\text {Agt1 }} \diamond_{\text {Tim }} F_{2}$ indicates that it is true in a situation that an agent knows the fact $F_{2}$ at a time. The expressions require development of a sound reasoning mechanism based on the semantics over many-dimensional worlds that the above approaches do not support.

Rigidity [30] is defined as a meta-property of concepts; a sort is rigid if the property is essential to all its instances in any time, space, belief, or situation, otherwise it is non-rigid. Let us assume many modal operators $\square_{1}, \ldots, \square_{m}, \diamond_{1}, \ldots, \diamond_{m}$ and common modal operators $\square, \diamond$. Intuitively, some of the modal operators are used to express temporal and situational operators $\square_{\text {Tim }}, \diamond_{\text {Tim }}, \square_{\text {Sit }}, \diamond_{\text {Sit }}$ and $k$ knowledge operators $\square_{\text {Agt1 } 1}, \ldots, \square_{\text {Agtk }}$ (for each knowledge of $k$ agents). In the semantics, the many modal operators $\square_{i}, \diamond_{i}$ are interpreted as specific modalities over time points, locations, or situations, and the common modal operators $\square$, are interpreted as general modalities over all the possible worlds. Given the temporal, situational, and $k$ knowledge operators, the common modal operator $\mathbf{\square}$ indicates the com- 


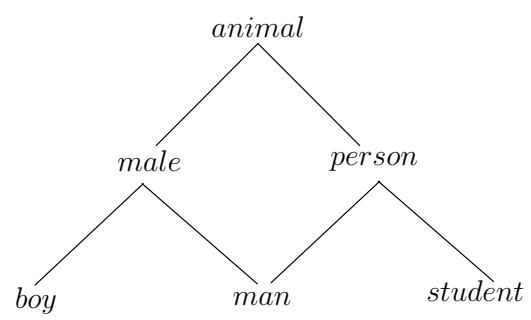

Figure 1: An example of sort-hierarchy

mon knowledge among $k$ agents in any time point and situation. That is, the modal formula $\square$ implies $\square_{\mathbf{T i m}} F \wedge \square_{\text {Sit }} F \wedge \square_{\text {Agt } 1} F \wedge \cdots \wedge \square_{\text {Agtk }} F$. In contrast, the standard common knowledge operator $\square_{\text {AllAgts }}$ only indicates the common knowledge among $k$ agents; i.e., the modal formula $\square_{\text {AllAgts }} F$ implies $\square_{\text {Agt1 }} F \wedge \cdots \wedge \square_{\text {Agtk }} F$. In order to handle relationships among time, situation, and knowledge worlds, accessibility relations over $n$-dimensional worlds are considered. For example, let $\left\langle t m, s t, w_{1}, \ldots, w_{k}\right\rangle$ be an $n$-dimensional world $(n=k+2)$ that indicates an $n$-tuple of time, situation, and $k$-agents' knowledge worlds. The modal formula $\square_{\text {Agt1 }} F$ is true if and only if for every world $w_{1}^{\prime}$ of agent 1 where $\vec{w}^{\prime}=\left\langle t m, s t, w_{1}^{\prime}, \ldots, w_{k}\right\rangle$ is accessible from the current world $\vec{w}=\left\langle t m, s t, w_{1}, \ldots, w_{k}\right\rangle, F$ is true in $\vec{w}^{\prime}$. Moreover, $\diamond_{\mathbf{T i m}} \square_{\mathbf{A g t 1}} F$ is true if and only if there exists some time $t m^{\prime}$ where $\vec{w}^{\prime \prime}=\left\langle t m^{\prime}, s t, w_{1}^{\prime}, \ldots, w_{k}\right\rangle$ is accessible from $\vec{w}^{\prime}=\left\langle t m, s t, w_{1}^{\prime}, \ldots, w_{k}\right\rangle$ and $F$ is true in $\vec{w}^{\prime \prime}$. For the purpose of our modeling of rigidity, we employ semantics of $n$-dimensional worlds different from the temporal logic formalisms in the area of agent systems, such as Alternating-time Temporal Logic (ATL) and Alternating-time Temporal Epistemic Logic (ATEL) [19].

We consider some examples of sorted modal formulas with the axiomatic system $S 4$ for the temporal and situational operators $\square_{\text {Tim }}$ and $\square_{\text {Sit }}$ and the common modal operator $\square_{\text {AllAgts }}$ and the axiomatic system $S 5$ for the $k$ knowledge operators $\square_{\mathrm{Agt1}}, \ldots, \square_{\mathrm{Agtk}}$. Let man, student, male, boy, person, and animal be sorts with the following subsort relation (sort hierarchy), as described in Figure 1:

$$
\begin{aligned}
\text { boy } & <\text { male } \\
\text { man } & <\text { male } \\
\text { man } & <\text { person } \\
\text { student } & <\text { person } \\
\text { person } & <\text { animal } \\
\text { male } & <\text { animal }
\end{aligned}
$$

The sorted formula man(john: person) is true at any time and situation if 
the sort man is rigid. In contrast, the sorted formula student(john: person) is true only in a particular situation if the sort student is not rigid. In the sort hierarchy, student(john: person) implies animal(john: person) because the anti-rigid sort student is a subsort of the rigid sort animal. This subsort relation is effectively defined in the rigidity of sorts where any rigid sort cannot be a subsort of an anti-rigid sort. In other words, some instances of each antirigid sort exist only in a particular time or situation that cannot belong to any rigid subsort.

According to the rigidity of sorts, many different modalities play an important role in representing anti-rigid sorts; i.e., a sortal property is true if it is dependent on time, space, belief, or situation. For example, the anti-rigidity of the sorted variable $x$ : person is represented by the sorted modal formula:

$$
\forall x: \operatorname{animal}\left(\diamond_{\operatorname{Tim}} \operatorname{child}(x: \operatorname{animal}) \wedge \diamond_{\operatorname{Tim}} \neg \operatorname{child}(x: \operatorname{animal})\right)
$$

which signifies that if $x$ is an animal there exists a time point in which he/she is a child, but there exists another time point in which he/she is not a child. Using the sort hierarchy, the formula implies more specific statements sorted by a subsort of animal, e.g.,

$$
\forall x: \operatorname{person}\left(\diamond_{\text {Tim }} \operatorname{child}(x: \text { person }) \wedge \diamond_{\operatorname{Tim}} \neg \operatorname{child}(x: \operatorname{person})\right)
$$

The rigidity of sorts also becomes more realistic when existential rigidity [29] is taken into account in ontological consideration. More precisely, a sort is existentially rigid if in any possible world for which an instance of the property exists, it instantiates the property. Consider the sorted modal formula:

$$
\forall x: \operatorname{person}\left(\square_{\text {Tim }} \operatorname{man}(x: \text { person })\right) .
$$

Under existential rigidity, this formula indicates that if $x$ is a person, then he is a man in the time period in which he exists. This statement logically derives the fact that there is no time point in which the person $x$ exists but he is not a man.

As a non-trivial example, the following sorted modal formulas are constructed by employing temporal and situational operators, $k$ knowledge operators, and common operators. By representing the positive introspection axiom (in the axiomatic systems for knowledge [10]), we can consider the sorted modal formula:

$$
\left(\square_{\mathbf{A g t 1} 1} \diamond_{\mathbf{S i t}} h a p p y(\text { bob: person })\right) \rightarrow\left(\square_{\mathbf{A g t 1} 1} \square_{\mathbf{A g t 1}} \diamond_{\mathbf{S i t}} h a p p y(\text { bob: person })\right)
$$

which states that an agent knows a fact which he/she knows, precisely, an agent knows the fact "Bob is happy in a situation where he is a person" when he/she 
knows that he/she knows it. Moreover, the negative introspection axiom (in the axiomatic systems for knowledge [10]) leads to the sorted modal formula:

$$
\left.\left(\neg \square_{\mathbf{A g t} \mathbf{1}} \diamond_{\mathbf{T i m}} \text { rich }(\text { bob }: \text { person })\right) \rightarrow\left(\square_{\mathbf{A g t 1}} \neg \square_{\mathbf{A g t 1}} \diamond_{\mathbf{T i m}} \text { rich(bob: person }\right)\right)
$$

This expresses the statement that an agent knows the fact "Bob is rich at a time point" which the agent does not know.

The common modal operator $\mathbf{a}$ can be used to represent a rigid sorted formula, i.e., a property is true in any time point, situation, or agent knowledge. For instance, we have a sorted modal formula for the rigid property male as follows:

$\mathbf{\square}_{\text {male }}($ bob: person $) \rightarrow\left(\square_{\mathbf{T i m}}\right.$ male $($ bob: person $) \wedge \square_{\text {Agt1 }}$ male $($ bob: person $\left.)\right)$

which says that if Bob is a male person, then he is a male person in any time point in which he exists and an agent knows the fact that he is a male person as long as he exists in the agent's mind (i.e., when the agent knows that Bob is alive). This axiom is validated by the feature of the common modal operator; i.e., the modal formula $\square$ implies $\square_{\text {Tim }} F \wedge \square_{\text {Sit }} F \wedge \square_{\text {Agt1 }} F \wedge \cdots \wedge \square_{\text {Agtk }} F$. In the modalities, the subsort relation male $<$ animal derives a more general statement as follows:

animal(bob: person)

$\left(\square_{\mathbf{T i m}}\right.$ animal $($ bob: person $) \wedge \square_{\mathbf{A g t 1}} \operatorname{animal}($ bob: person $\left.)\right)$

Using the subsort relation person < animal, another general statement can be derived as follows:

$$
\begin{aligned}
& (\exists x: \text { animal })(\square \text { male }(x: \text { animal }) \rightarrow \\
& \left.\left(\square_{\mathbf{T i m}} \text { male }(x: \text { animal }) \wedge \square_{\mathbf{A g t 1}} \text { male }(x: \text { animal })\right)\right)
\end{aligned}
$$

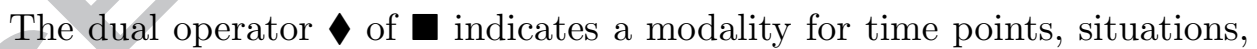
and $k$ agents' knowledge, which can represent a non-rigid sorted formula (i.e., a property is true in a time point, situation, or agent knowledge). As an example of this, we can consider a sorted modal formulas for the non-rigid property child as follows:

$$
\diamond_{\text {Tim }} \operatorname{child}(\text { bob }: \text { person }) \rightarrow{ }^{\prime} \operatorname{child}(\text { bob }: \text { person })
$$

which states that if Bob is a child in a time point, then the fact that Bob is a child is true in another time point, situation, or agent knowledge.

These examples motivate us to enhance order-sorted logic by incorporating many modalities under existential rigidity.

First-order modal logics have long been investigated independently from order-sorted logic. Garson [8] discussed different systems for variants of quantified modal logics. Fitting and Mendelsohn [5] treated the rigidity of terms and 
constant/varying domains by means of a tableau calculus and predicate abstraction. Cialdea-Mayer and Cerrito [2] proposed a prefixed tableau calculus for all variants of quantified modal logics with respect to cumulative/varying domains, rigid/non-rigid terms, and local/non-local terms. However, the existing approaches do not provide a combination of existential rigidity and many modalities in logic. In particular, there is no reasoning system for the integrated logic of sorted expressions, rigidity, and many modalities. To achieve the reasoning system for the combination, additional inference rules for the interaction between sorts and modalities, which cannot be obtained by simply combining them, have to be defined.

Based on the above motivation, we study an extension of order-sorted logic by introducing existential rigidity and many modalities into sorted terms and formulas. The existential rigidity and non-rigidity of sorts are expressed by the distinctions among rigid and anti-rigid sorted terms together with an existential predicate. That is, many different modal operators are used to express the non-rigidity of sorts in various possible worlds such as time, space, belief, or situation. In the semantic definition, sorted modal formulas are interpreted over the Cartesian product of sets of possible worlds.

In order to apply our extension to reasoning mechanisms, we propose a labeled tableau calculus that tests the satisfiability and validity of sorted modal formulas. This calculus is obtained by extending the prefixed tableau calculus proposed by Cialdea-Mayer and Cerrito. In the derivation process where decomposed formulas are derived by rule application in calculus, sorted modal formulas are labeled by a pair of the type of a world and the world itself. New inference rules (many modal operator, sorted quantifier, rigid/anti-rigid sort predicate, and existential predicate rules) are included to handle sorted expressions and many modalities supporting existential rigidity.

The rest of this paper is arranged as follows. Section 2 formalizes the syntax and semantics of order-sorted modal logic. In Section 3, we discuss a labeled tableau calculus for our proposed order-sorted modal logic, which enables us to check the satisfiability and validity of sorted modal formulas. In Section 4, we show the completeness of the labeled tableau calculus. Then, we give some examples for testing the validity of sorted modal formulas. Finally, in Section 5, we provide our conclusion.

\section{Order-Sorted Modal Logic}

We define the syntax and semantics of order-sorted logic with many modalities and existential rigidity. 


\subsection{Syntax}

The alphabet of a sorted first-order modal language $\mathcal{L}$ with rigidity and sort predicates comprises the following symbols: a countable set $\mathcal{T}$ of type symbols as rigid sorts (including the greatest type $\top$ ), countable set $\mathcal{S}_{\mathcal{A}}$ of antirigid sort symbols $\left(\mathcal{T} \cap \mathcal{S}_{\mathcal{A}}=\emptyset\right)$, countable set $C$ of constant symbols, countable set $F_{n}$ of $n$-ary function symbols for each natural number $n$, and countable set $P_{n}$ of $n$-ary predicate symbols for each natural number $n$ with the existential predicate symbol $E$ and the set $P_{\mathcal{T} \cup \mathcal{S}_{\mathcal{A}}}$ of sort predicate symbols $\left\{p_{s} \mid s \in \mathcal{T} \cup \mathcal{S}_{\mathcal{A}}\right\}$, the connectives $\wedge, \vee, \rightarrow, \neg$, quantifiers $\forall, \exists$, modal operators $\left\{\square_{1}, \ldots, \square_{m}\right\},\left\{\diamond_{1}, \ldots, \diamond_{m}\right\}, \boldsymbol{\square}, \diamond$ with a natural number $m$, and auxiliary symbols (,). We write $F=\bigcup_{n \geq 0} F_{n}$ and $P=\bigcup_{n \geq 0} P_{n}$.

All type symbols $\tau$ and anti-rigid sort symbols $\sigma$ are called sort symbols $s$. $\mathcal{T} \cup \mathcal{S}_{\mathcal{A}}$ is the set of sort symbols. $V_{s}$ denotes an infinite set of variables $x_{s}$ of sort $s$. We abbreviate variables $x_{\top}$ of sort $\top$ as $x$. The set of variables of all sorts is denoted by $V=\bigcup_{s \in \mathcal{T} \cup \mathcal{S}_{\mathcal{A}}} V_{s}$. The unary predicates $p_{s} \in P_{1}$ indexed by the sorts $s$ (called sort predicates) are introduced for all sorts $s \in \mathcal{T} \cup \mathcal{S}_{\mathcal{A}}$. In particular, the predicate $p_{\tau}$ indexed by a type $\tau$ is called a type predicate, and the predicate $p_{\sigma}$ indexed by an anti-rigid sort $\sigma$ is called an anti-rigid sort predicate. Hereinafter, we assume that every sorted first-order modal language $\mathcal{L}$ contains all the sort predicates in $P_{\mathcal{T} \cup \mathcal{S}_{\mathcal{A}}}$.

Let $T \subseteq \mathcal{T}$ and $S_{A} \subseteq \mathcal{S}_{\mathcal{A}}$. A constant declaration is of the form $c: \rightarrow \tau$ if $c \in C$ and $\tau \in T$. A function declaration is of the form $f: \tau_{1} \times \cdots \times \tau_{n} \rightarrow \tau$ if $f \in F_{n}(n>0)$ and $\tau_{1}, \ldots, \tau_{n}, \tau \in T$. A predicate declaration $p: s_{1} \times \cdots \times s_{n}$ if $p \in P_{n}$ and $s_{1}, \ldots, s_{n} \in T \cup S_{A}$. In particular, for each type or anti-rigid sort predicate $p_{s} \in P_{T \cup S_{A}}$, the predicate declaration is of the form $p_{s}: T$ where $T$ is the greatest type.

Definition 1 (Sorted Signatures) A signature of a sorted first-order modal language $\mathcal{L}$ with rigidity and sort predicates (called sorted signature) is a tuple $\Sigma=\left(T, S_{A}, \leq, \Omega\right)$ such that

1. $\left(T \cup S_{A}, \leq\right)$ is a partially ordered set of sorts where $T \cup S_{A}$ is the union of a set of type symbols and a set of anti-rigid sort symbols in $\mathcal{L}$ and each ordered pair $s_{i} \leq s_{j}$ is a subsort relation (i.e., $s_{i}$ is a subsort of $s_{j}$ ).

2. no rigid sort is a subsort of an anti-rigid sort.

3. $\Omega$ is a set of constant, function, and predicate declarations.

Intuitively, the distinction between rigid and anti-rigid sorts is given as follows. If an entity has a rigid property, then it must have that property in any possible world. By following it, a sort is categorized as rigid if every instance of the sort is an instance of that in any possible world. Otherwise, it is anti-rigid. 
Constants and functions are required to be rigidly sorted in order to avoid the anti-rigid domains and ranges of constants and functions. The sort declarations of constants $c$ and functions $f$ are therefore denoted by the forms $c: \rightarrow \tau$ and $f: \tau_{1} \times \cdots \times \tau_{n} \rightarrow \tau$ where types $\tau_{i}, \tau$ are used for the declarations. By contrast, predicates may be anti-rigid. The sort declarations of predicates are denoted by the form $p: s_{1} \times \cdots \times s_{n}$ where types and anti-rigid sorts $s_{i}$ can be used to set the domains of the predicates $p$.

In [14], the reason for not allowing the anti-rigid sorts of constants and functions has been explained. We consider the following declarations of unrigidly sorted constants, functions, and predicates:

$$
\begin{aligned}
& \text { john: } \rightarrow \text { student, } \\
& \text { father }: \text { person } \rightarrow \text { teacher, } \\
& \text { getting_a_scholarship }: \text { student. }
\end{aligned}
$$

Unfortunately, the anti-rigid sorts student and teacher give rise to the sorted terms:

$$
\begin{aligned}
& \text { john: student, } \\
& \text { father(john: student }): \text { teacher, } \\
& \text { getting_a_scholarship }\left(x_{\text {student }}\right) \text {. }
\end{aligned}
$$

Due to the anti-rigidity of student and teacher, John is not a student in some possible worlds and John's father is not a teacher in some possible worlds. These expressions are regarded as ill-sorted errors in some possible worlds. On the contrary, the anti-rigid sort of the predicate leads to $g e t t i n g \_a \_s c h o l a r s h i p\left(x_{\text {student }}\right)$ being true or false but it causes no ill-sorted error.

In the sorted signature, the three types of terms: typed term, anti-rigid sorted term, and sorted term are inductively defined in a sorted first-order modal language $\mathcal{L}_{\Sigma}$.

Definition 2 (Typed Terms) Let $\Sigma=\left(T, S_{A}, \leq, \Omega\right)$ be a sorted signature. The set $\mathcal{T}_{\tau}^{-}$of terms of type $\tau$ (called typed terms) is the smallest set such that

1. for every $x_{\tau} \in V_{\tau}, x_{\tau} \in \mathcal{T}_{\tau}^{-}$;

2. for every $c \in C$ with $c$ : $\rightarrow \tau \in \Omega, c_{\tau} \in \mathcal{T}_{\tau}^{-}$;

3. if $t_{1} \in \mathcal{T}_{\tau_{1}}^{-}, \ldots, t_{n} \in \mathcal{T}_{\tau_{n}}^{-}, f \in F_{n}$, and $f: \tau_{1} \times \cdots \times \tau_{n} \rightarrow \tau \in \Omega$, then $f_{\tau^{*}, \tau}\left(t_{1}, \ldots, t_{n}\right) \in \mathcal{T}_{\tau}^{-}$with $\tau^{*}=\tau_{1}, \ldots, \tau_{n}$

4. if $t \in \mathcal{T}_{\tau^{\prime}}^{-}$and $\tau^{\prime} \leq \tau$, then $t \in \mathcal{T}_{\tau}^{-}$.

Definition 3 (Anti-Rigid Sorted Terms) Let $\Sigma=\left(T, S_{A}, \leq, \Omega\right)$ be a sorted signature. The set $\mathcal{T}_{\sigma}^{-}$of terms of anti-rigid sort $\sigma$ (called anti-rigid sorted terms) is the smallest set such that 
1. for every $x_{\sigma} \in V_{\sigma}, x_{\sigma} \in \mathcal{T}_{\sigma}^{-}$;

2. if $t \in \mathcal{T}_{\sigma^{\prime}}^{-}$and $\sigma^{\prime} \leq \sigma$, then $t \in \mathcal{T}_{\sigma}^{-}$.

The anti-rigid sorted terms are only variables because the anti-rigid sorts of constants and functions are not allowed in the sorted signatures (as explained above).

Definition 4 (Sorted Terms) Let $\Sigma=\left(T, S_{A}, \leq, \Omega\right)$ be a sorted signature. The set $\mathcal{T}_{s}$ of terms of sort $s$ (called sorted terms) is the smallest set such that

1. if $s=\tau$, then $\mathcal{T}_{s}^{-} \subseteq \mathcal{T}_{s}$;

2. if $t \in \mathcal{T}_{s^{\prime}}$ and $s^{\prime} \leq s$, then $t \in \mathcal{T}_{s}$.

We denote the set of ground terms of sort $s$ by $\mathcal{T}_{s, 0}$. Based on the rigidity of types and anti-rigid sorts, any anti-rigid sorted term (in $\mathcal{T}_{\sigma}^{-}$) must be a variable term whereas typed terms (in $\mathcal{T}_{\tau}^{-}$) can contain constants and functions. In other words, every anti-rigid sorted term is not rigid (e.g., $x_{\text {student }}$ ) and every typed term is rigid (e.g., $c_{\text {person }}$ ). We define $\operatorname{sort}(t)$ as the sort of a term $t$; precisely, we define $\operatorname{sort}(t)=s$ if $t$ is of the form $x_{s}, c_{s}$, or $f_{\tau^{*}, s}\left(t_{1}, \ldots, t_{n}\right)$.

Next, the set of sorted modal formulas in the language $\mathcal{L}_{\Sigma}$ is given as follows.

Definition 5 (Sorted Modal Formulas) Let $\Sigma=\left(T, S_{A}, \leq, \Omega\right)$ be a sorted signature. The set $\mathcal{F}$ of formulas is the smallest set such that

1. if $t_{1} \in \mathcal{T}_{s_{1}}, \ldots, t_{n} \in \mathcal{T}_{s_{n}}, p \in P_{n}$, and $p: s_{1} \times \cdots \times s_{n} \in \Omega$, then $p\left(t_{1}, \ldots, t_{n}\right)$ is a formula;

2. if $t \in \mathcal{T}_{\tau}, p_{s} \in P_{T \cup S_{A}}$, and $p_{s}: \top \in \Omega$, then $p_{s}(t)$ is a formula;

3. if $t \in \mathcal{T}_{\text {T }}$, then $E(t)$ is a formula;

4. if $F, F_{1}$, and $F_{2}$ are formulas, then $\neg F,\left(\forall x_{s}\right) F,\left(\exists x_{s}\right) F, \square_{i} F, \diamond_{i} F, \boldsymbol{\square} F$, $\checkmark F, F_{1} \wedge F_{2}, F_{1} \vee F_{2}$, and $F_{1} \rightarrow F_{2}$ are formulas.

The existential predicate formula $E(t)$ asserts the existence of an individual denoted by a term $t$ in a possible world. A sorted formula is called closed if it does not contain free variables. We use some of the modal operators to represent temporal and situational operators $\square_{\text {Tim }}, \diamond_{\text {Tim }}, \square_{\text {Sit }}, \diamond_{\text {Sit }}$ and $k$ knowledge operators $\square_{\text {Agt1 }}, \ldots, \square_{\text {Agtk }}$ (for each knowledge of $k$ agents). We abbreviate $\left(\square_{\text {Agt1 }} F\right) \wedge \cdots \wedge\left(\square_{\text {Agtk }} F\right)$ by the common knowledge operator $\square_{\text {AllAgts }} F$. 


\subsection{Semantics}

Here we define the semantics of our sorted first-order modal languages $\mathcal{L}_{\Sigma}$. Let $w_{1}, \ldots, w_{n}$ be worlds. In the following, we denote an $n$-tuple of worlds $\left\langle w_{1}, \ldots, w_{n}\right\rangle$ by $\vec{w}$.

Definition 6 (Sorted $\Sigma$-Structures) Let $\Sigma$ be a sorted signature. A sorted $\Sigma$-structure $M$ is a tuple $\left(W, \vec{w}_{0},\left\{R_{1}, \ldots, R_{m}\right\}, R, U, I\right)$ such that

1. $W=W_{1} \times \cdots \times W_{m}$ where each $W_{i}$ is a non-empty set of worlds and for every $1 \leq i<j \leq m, W_{i} \cap W_{j}=\emptyset$;

2. $\vec{w}_{0} \in W$;

3. $R_{i} \subseteq\left\{\left\langle\left\langle w_{1}, \ldots, w_{m}\right\rangle,\left\langle w_{1}^{\prime}, \ldots, w_{m}^{\prime}\right\rangle\right\rangle \in W \times W \mid \forall k \in\{1, \ldots, m\} \backslash\{i\}\left(w_{k}=\right.\right.$ $\left.\left.w_{k}^{\prime}\right)\right\}$ where $R_{i}$ is reflexive and transitive, or reflexive, transitive, and symmetric;

4. $R \subseteq W \times W$ is a superset of $R_{1} \cup \cdots \cup R_{m}$ where $R$ is reflexive and transitive;

5. $U_{\vec{w}}$ is a non-empty set of individuals for each $\vec{w} \in W$;

6. $U$ is a superset of $\bigcup_{\vec{w} \in W} U_{\vec{w}}$;

7. $I=\left\{I_{\vec{w}} \mid \vec{w} \in W\right\}$ is the set of interpretation functions $I_{\vec{w}}$ for all tuples $\vec{w} \in W$ with the following conditions:

(a) if $s \in T \cup S_{A}$, then $I_{\vec{w}}(s) \subseteq U_{\vec{w}}$, (in particular, if $s=\top$, then $\left.I_{\vec{w}}(s)=U_{\vec{w}}\right)$,

(b) if $s_{i} \leq s_{j}$ with $s_{i}, s_{j} \in T \cup S_{A}$, then $I_{\vec{w}}\left(s_{i}\right) \subseteq I_{\vec{w}}\left(s_{j}\right)$,

(c) if $c \in C$ and $c: \rightarrow \tau \in \Omega$, then $I_{\vec{w}}(c) \in I_{\vec{w}}(\tau)$,

(d) if $f \in F_{n}$ and $f: \tau_{1} \times \cdots \times \tau_{n} \rightarrow \tau \in \Omega$, then $I_{\vec{w}}(f): I_{\vec{w}}\left(\tau_{1}\right) \times \cdots \times$ $I_{\vec{w}}\left(\tau_{n}\right) \rightarrow I_{\vec{w}}(\tau)$,

(e) if $p \in P_{n}$ and $p: s_{1} \times \cdots \times s_{n} \in \Omega$, then $I_{\vec{w}}(p) \subseteq I_{\vec{w}}\left(s_{1}\right) \times \cdots \times I_{\vec{w}}\left(s_{n}\right)$, and

(f) if $s \in T \cup S_{A}$ and $p_{s} \in P_{T \cup S_{A}}$, then $I_{\vec{w}}(s)=I_{\vec{w}}\left(p_{s}\right)$.

In the sorted $\Sigma$-structures, an individual set $U_{\vec{w}}$ for each $n$-tuple $\vec{w}$ of worlds is used to represent the set of individuals that exist in an $n$-dimensional world $\vec{w}=\left\langle w_{1}, \ldots, w_{n}\right\rangle$. The accessibility relation $\left\langle\left\langle w_{1}, \ldots, w_{m}\right\rangle,\left\langle w_{1}^{\prime}, \ldots, w_{m}^{\prime}\right\rangle\right\rangle \in R_{i}$ indicates that $\left\langle w_{1}^{\prime}, \ldots, w_{m}^{\prime}\right\rangle$ is accessible from $\left\langle w_{1}, \ldots, w_{m}\right\rangle$ such that $w_{k}=w_{k}^{\prime}$ for every $k \in\{1, \ldots, m\} \backslash\{i\}$. Unlike the general accessibility $R$, accessibility $R_{i}$ indexed by $i$ is limited to a relation that accesses $w_{i}$ in multiple dimensional worlds of $\left\langle w_{1}, \ldots, w_{m}\right\rangle$. 
We define the existential rigidity of sorts, constants, and functions in sorted $\Sigma$-structures by supporting the conditions of individual existence in the following manner.

Definition 7 (Existential Rigidity) Let $M=\left(W, \vec{w}_{0},\left\{R_{1}, \ldots, R_{m}\right\}, R, U, I\right)$ be a sorted $\Sigma$-structure, $\vec{w} \in W, I_{\vec{w}}$ be an interpretation function for $\vec{w}, d \in U_{\vec{w}}$, and let $R$ be an accessibility relation over $W \times W$. Then, $M$ is a sorted $\Sigma-$ structure with existential rigidity if for all $\vec{w}_{i}, \vec{w}_{j} \in W$ and for any interpretation functions $I_{\vec{w}_{i}}$ and $I_{\vec{w}_{j}}$ for $\vec{w}_{i}$ and $\vec{w}_{j}$, the following conditions hold:

1. if $I_{\vec{w}_{i}}(c), I_{\vec{w}_{j}}(c) \in U_{\vec{w}_{i}} \cap U_{\vec{w}_{j}}$, then $I_{\vec{w}_{i}}(c)=I_{\vec{w}_{j}}(c)$ where c is a constant;

2. for any $d_{1}, \ldots, d_{n} \in U_{\vec{w}_{i}} \cap U_{\vec{w}_{j}}$, if $\left\{I_{\vec{w}_{i}}(f)\left(d_{1}, \ldots, d_{n}\right), I_{\vec{w}_{j}}(f)\left(d_{1}, \ldots, d_{n}\right)\right\} \subseteq$ $U_{\vec{w}_{i}} \cap U_{\vec{w}_{j}}$, then $I_{\vec{w}_{i}}(f)\left(d_{1}, \ldots, d_{n}\right)=I_{\vec{w}_{j}}(f)\left(d_{1}, \ldots, d_{n}\right)$ where $f$ is an $n$ ary function;

3. for every type $\tau$, if $d \in I_{\vec{w}}(\tau)$ and $\left\langle\vec{w}, \vec{w}^{\prime}\right\rangle \in R$, then $d \in U_{\vec{w}^{\prime}}$ implies $d \in I_{\vec{w}^{\prime}}(\tau)$

4. for every anti-rigid sort $\sigma$, if $d \in I_{\vec{w}}(\sigma)$, then there exists $\vec{w}_{j} \in W$ with $\left\langle\vec{w}, \vec{w}_{j}\right\rangle \in R$ such that $d \notin I_{\vec{w}_{j}}(\sigma)$ with $d \in U_{\vec{w}_{j}}$.

In order to support the existential rigidity of sorts, the interpretation function and accessibility relation in sorted $\Sigma$-structures with existential rigidity (of Definition 7 ) is restricted by adding some conditions to them in sorted $\Sigma$ structures (of Definition 6).

The denotation of terms is defined by introducing the set $C_{U}$ of new constants $\bar{d}$ for individuals $d$ in $U$ where every new constant is interpreted by itself. In what follows, we adopt a sorted first-order modal language $\mathcal{L}_{\Sigma}$ extended by adding the set $C_{U}$ of new constants.

Definition 8 Let $M=\left(W, \vec{w}_{0},\left\{R_{1}, \ldots, R_{m}\right\}, R, U, I\right)$ be a sorted $\Sigma$-structure and let $\vec{w} \in W$. The denotation $\llbracket \rrbracket_{\vec{w}}: \mathcal{T}_{\top, 0} \rightarrow U_{\vec{w}}$ is defined by the following rules:

1. $\llbracket c_{\tau} \rrbracket_{\vec{w}}=I_{\vec{w}}(c)$ for $c \in C$ where $c: \rightarrow \tau \in \Omega$,

2. $\llbracket \bar{d} \rrbracket_{\vec{w}}=d$ for $\bar{d} \in C_{U}$, and

3. $\llbracket f_{\tau^{*}, \tau}\left(t_{1}, \ldots, t_{n}\right) \rrbracket_{\vec{w}}=I_{\vec{w}}(f)\left(\llbracket t_{1} \rrbracket_{\vec{w}}, \ldots, \llbracket t_{n} \rrbracket_{\vec{w}}\right)$ for $f \in F_{n}$ where $f: \tau_{1} \times$ $\cdots \times \tau_{n} \rightarrow \tau \in \Omega$.

Note that $\mathcal{T}_{\top, 0}$ (the domain of $\llbracket \rrbracket_{\vec{w}}$ ) denotes the set of ground terms of all sorts since $\top$ is the greatest type and $\mathcal{T}_{s, 0}$ denotes the set of ground terms of sort $s$ and all its subsorts. A closed formula is a sorted modal formula without free variables. We define the set of subterms of a term $t$ as follows: 
1. if $t=c_{\tau}$, then $\operatorname{sub}(t)=\left\{c_{\tau}\right\}$;

2. if $t=f_{\tau^{*}, \tau}\left(t_{1}, \ldots, t_{n}\right): s$, then $s u b(t)=\left\{f_{\tau^{*}, \tau}\left(t_{1}, \ldots, t_{n}\right): s\right\} \cup \operatorname{sub}\left(t_{1}\right) \cup$ $\cdots \cup \operatorname{sub}\left(t_{n}\right)$.

To define the satisfiability of sorted modal formulas, the existence of individuals denoted by terms in each world is handled. Let $M=\left(W, \vec{w}_{0},\left\{R_{1}, \ldots, R_{m}\right\}\right.$, $R, U, I)$ be a sorted $\Sigma$-structure, let $F, F_{1}$, and $F_{2}$ be sorted modal formulas, let $\vec{w} \in W$, and let $\llbracket t \rrbracket_{\vec{w}}$ be the denotation of a sorted ground term $t$ in $\vec{w}$. The set $N e x_{\vec{w}}$ of closed formulas with sorted ground terms non-existing in $\vec{w}$ is the smallest set such that

1. $p\left(t_{1}, \ldots, t_{n}\right) \in N e x_{\vec{w}}$ iff for some ground term $t \in \operatorname{sub}\left(t_{1}\right) \cup \cdots \cup \operatorname{sub}\left(t_{n}\right)$, $\llbracket t \rrbracket_{\vec{w}} \notin U_{\vec{w}}$

2. $\neg F,\left(\forall x_{s}\right) F,\left(\exists x_{s}\right) F \in N e x_{\vec{w}}$ iff $F \in N e x_{\vec{w}}$;

3. $\square_{i} F, \diamond_{i} F, \boldsymbol{\square} F, \diamond F \notin N e x_{\vec{w}}$;

4. $F_{1} \wedge F_{2} \in N e x_{\vec{w}}$ iff $F_{1} \in N e x_{\vec{w}}$ or $F_{2} \in N e x_{\vec{w}}$;

5. $F_{1} \vee F_{2} \in N e x_{\vec{w}}$ iff $F_{1} \in N e x_{\vec{w}}$ and $F_{2} \in N e x_{\vec{w}}$;

6. $F_{1} \rightarrow F_{2} \in N e x_{\vec{w}}$ iff $\neg F_{1} \in N e x_{\vec{w}}$ and $F_{2} \in N e x_{\vec{w}}$.

Definition 9 ( $\Sigma$-Satisfiability Relation) Let $M=\left(W, \vec{w}_{0},\left\{R_{1}, \ldots, R_{m}\right\}, R\right.$, $U, I)$ be a $\Sigma$-structure, let $F$ be a closed formula, and let $\vec{w}=\left\langle w_{1}, \ldots, w_{n}\right\rangle \in W$. The $\Sigma$-satisfiability relation $\vec{w}=F$ is defined inductively as follows:

1. $\vec{w}=p\left(t_{1}, \ldots, t_{n}\right)$ iff $\left(\llbracket t_{1} \rrbracket_{\vec{w}}, \ldots, \llbracket t_{n} \rrbracket_{\vec{w}}\right) \in I_{\vec{w}}(p)$;

2. $\vec{w} \models E(t)$ iff there exists $d \in U_{\vec{w}}$ such that $\llbracket t \rrbracket_{\vec{w}}=d$;

3. $\vec{w}=\neg F$ iff $\vec{w} \mid \neq F$;

4. $\vec{w} \models F_{1} \wedge F_{2}$ iff $\vec{w}=F_{1}$ and $\vec{w}=F_{2}$;

5. $\vec{w} \models F_{1} \vee F_{2}$ iff $\vec{w}=F_{1}$ or $\vec{w}=F_{2}$;

6. $\vec{w} \models F_{1} \rightarrow F_{2}$ iff $\vec{w} \mid \neq F_{1}$ or $\vec{w}=F_{2}$;

7. $\vec{w} \models\left(\forall x_{s}\right) F$ iff for all $d \in I_{\vec{w}}(s), \vec{w} \models F\left[x_{s} / \bar{d}\right]$;

8. $\vec{w} \models\left(\exists x_{s}\right) F$ iff for some $d \in I_{\vec{w}}(s), \vec{w}=F\left[x_{s} / \bar{d}\right]$;

9. $\vec{w} \models \square_{i} F$ iff for all $w^{\prime} \in W_{i}$ with $\left\langle\vec{w}, \vec{w}^{\prime}\right\rangle \in R_{i}, \vec{w}^{\prime} \models F$ or $F \in N e x_{\vec{w}^{\prime}}$ where $\vec{w}^{\prime}=\left\langle w_{1}, \ldots, w_{i-1}, w^{\prime}, w_{i+1}, \ldots, w_{n}\right\rangle$; 
10. $\vec{w}=\diamond_{i} F$ iff for some $w^{\prime} \in W_{i}$ with $\left\langle\vec{w}, \vec{w}^{\prime}\right\rangle \in R_{i}, \vec{w}^{\prime}=F$ and $F \notin N e x_{\vec{w}^{\prime}}$ where $\vec{w}^{\prime}=\left\langle w_{1}, \ldots, w_{i-1}, w^{\prime}, w_{i+1}, \ldots, w_{n}\right\rangle$;

11. $\vec{w}=\mathbf{\square} F$ iff for all $\vec{w}^{\prime} \in W$ with $\left\langle\vec{w}, \vec{w}^{\prime}\right\rangle \in R, \vec{w}^{\prime}=F$ or $F \in N e \vec{w}_{\vec{w}^{\prime}}$;

12. $\vec{w} \models \nabla$ iff for some $\vec{w}^{\prime} \in W$ with $\left\langle\vec{w}, \vec{w}^{\prime}\right\rangle \in R, \vec{w}^{\prime}=F$ and $F \notin N e x_{\vec{w}^{\prime}}$.

The modal formula $\square_{i} F$ (or $\boldsymbol{\square} F$ ) is satisfied in a world $\vec{w}$ if for any world $\vec{w}^{\prime}$ accessible from $\vec{w}, F$ is satisfied in $\vec{w}^{\prime}\left(\vec{w}^{\prime} \models F\right)$ or some ground terms in $F$ do not exist in $\vec{w}^{\prime}\left(F \in N e x_{\vec{w}^{\prime}}\right)$. Let $F$ be a formula. It is $\Sigma$-true in $M$ if $\vec{w}_{0}=F$ ( $M$ is a $\Sigma$-model of $F$ ). If $F$ has a $\Sigma$-model, it is $\Sigma$-satisfiable, otherwise, it is $\Sigma$-unsatisfiable. $F$ is $\Sigma$-valid if every sorted $\Sigma$-structure is a $\Sigma$-model of $F$. Let $\Psi$ be a set of formulas. A formula $F$ is a consequence of $\Psi$ in the class of $\Sigma$-structures (denoted $\Psi \models F$ ) if for every $\Sigma$-structure $M, M \models \Psi$ implies $M \models F$.

To test the satisfiability of any closed formula, the following proposition guarantees that any closed formula can be transformed into an equivalent one in negation normal form (i.e., negation occurs only in front of an atomic formula). Let $F_{1}$ and $F_{2}$ be closed formulas. The formulas $F_{1}$ and $F_{2}$ are semantically equivalent (denoted $F_{1} \simeq F_{2}$ ) if for every sorted $\Sigma$-structure with existential rigidity $M=\left(W, \vec{w}_{0},\left\{R_{1}, \ldots, R_{m}\right\}, R, U, I\right)$ and for every $\vec{w} \in W, \vec{w} \models F_{1}$ if and only if $\vec{w}=F_{2}$.

Proposition 1 Let $F, F_{1}$, and $F_{2}$ be closed formulas and let $i \in\{1, \ldots, m\}$. The following semantic equivalences hold:

$$
\begin{aligned}
\neg \neg F & \simeq F \\
\neg\left(F_{1} \wedge F_{2}\right) & \simeq \neg F_{1} \vee \neg F_{2} \\
\neg\left(F_{1} \vee F_{2}\right) & \simeq \neg F_{1} \wedge \neg F_{2} \\
\neg\left(F_{1} \rightarrow F_{2}\right) & \simeq F_{1} \wedge \neg F_{2} \\
\neg\left(\forall x_{s}\right) F & \simeq\left(\exists x_{s}\right) \neg F \\
\neg\left(\exists x_{s}\right) F & \simeq\left(\forall x_{s}\right) \neg F \\
\neg \mathbf{\square} F & \simeq{ }^{\prime}(\neg F) \\
\neg \vee F & \simeq \mathbf{\square}(\neg F) \\
\neg \square_{i} F & \simeq \diamond_{i}(\neg F) \\
\neg \diamond_{i} F & \simeq \square_{i}(\neg F)
\end{aligned}
$$

Proof. Let $M=\left(W, \vec{w}_{0},\left\{R_{1}, \ldots, R_{m}\right\}, R, U, I\right)$ be any sorted $\Sigma$-structure with existential rigidity and let $\vec{w} \in W$. By Definition 9 , the semantic equivalences can be proved as follows: $(\neg \neg F \simeq F) \vec{w} \models \neg \neg F$ if and only $\vec{w} \mid \neq \neg F$ if and only if $\vec{w} \models F . \quad(\neg F \simeq \mathbf{\square}(\neg F)) \vec{w} \models \neg F$ if and only if for all $\vec{w}^{\prime} \in W$ with $\left\langle\vec{w}, \vec{w}^{\prime}\right\rangle \in R, \vec{w}^{\prime} \mid \neq F$ or $F \in N e x_{\vec{w}^{\prime}}$ if and only if for all $\vec{w}^{\prime} \in W$ with $\left\langle\vec{w}, \vec{w}^{\prime}\right\rangle \in R$, 
$\vec{w}^{\prime} \models \neg F$ or $\neg F \in N e x_{\vec{w}^{\prime}}$ if and only if $\vec{w}=\mathbf{\square} \neg F .\left(\neg \square_{i} F \simeq \diamond_{i}(\neg F)\right) \vec{w}=\neg \square_{i} F$ if and only if for some $\vec{w}^{\prime} \in W_{i}$ with $\left\langle\vec{w}, \vec{w}^{\prime}\right\rangle \in R_{i}, \vec{w}^{\prime} \mid \neq F$ and $F \notin N e x_{\vec{w}^{\prime}}$ if and only if for some $\vec{w}^{\prime} \in W_{i}$ with $\left\langle\vec{w}, \vec{w}^{\prime}\right\rangle \in R_{i}, \vec{w}^{\prime}=\neg F$ and $\neg F \notin N e x_{\vec{w}^{\prime}}$ if and only if $\vec{w}=\diamond_{i}(\neg F)$. Similarly, the other cases can be shown.

\section{Tableau Calculus}

In this section, we present a labeled tableau calculus for testing the satisfiability of a sorted modal formula in order-sorted modal logic.

Let $A$ be a closed formula in negation normal form (i.e., negation occurs only in front of an atomic formula) and $\Psi$ be a finite set of closed formulas in negation normal form. We define the annotated term $t^{(i, n)}$ by annotating each constant symbol and function symbol with $(i, n) \in\left\{W, T_{1}, \ldots, T_{m}\right\} \times \mathrm{N}$ (e.g., $c_{\tau}^{(i, n)}$ and $\left.f_{\tau_{1}, \tau_{2}, \tau}^{(i, n)}\left(x_{\tau_{1}}, c_{\tau_{2}}^{(i, n)}\right)\right)$. The annotated set $\Psi^{(i, n)}$ and formula $A^{(i, n)}$ are obtained as follows: If $\Psi=\left\{A_{1}, \ldots, A_{k}\right\}$ then $\Psi^{(i, n)}=\left\{A_{1}^{(i, n)}, \ldots, A_{k}^{(i, n)}\right\}$. If $A_{i}=p\left(t_{1}, \ldots, t_{k}\right)$ then $A_{i}^{(i, n)}=p\left(t_{1}^{(i, n)}, \ldots, t_{k}^{(i, n)}\right)$, and otherwise, $A_{i}^{(i, n)}=A_{i}$. The annotated term $t^{(i, n)}$ implies that it exists in the world corresponding to $(i, n)$. Let $W$ and $T_{i}$ denote the two types of worlds corresponding to $\boldsymbol{\square}$ and $\square_{i}$, respectively. Each node in a tableau is labeled with a formula set $(i, n): \Psi$ where $i \in\left\{W, T_{1}, \ldots, T_{m}\right\}$ and $n \in \mathrm{N}$. The initial tableau for $\Psi$ is the single node $(W, 0): S^{(W, 0)}$.

The tableau calculus contains conjunction and disjunction rules, existential predicate rules, modal operator rules, sorted quantifier rules, and sort predicate rules. The pair $(i, n)$ of labels $i$ and $n$ denotes a type of worlds and a natural number. Note that in order to support many modalities, modal formulas are labeled by a pair $(i, n)$ such that two labels $i$ and $n$ are needed to represent the type of a current world $i$ and the level of a current modality $n$. For example, consider the modal formula $F=\diamond_{\text {Sit }} \diamond_{\text {Tim }}$ rich(bob: person) that is decomposed in the inference process as follows:

$$
\frac{\left(\text { W,0): } \diamond_{\text {Sit }} \diamond_{\text {Tim }} \text { rich(bob: person }\right)}{\frac{(\text { Sit }, 1): \diamond_{\operatorname{Tim}} \text { rich }(\text { bob: person })}{\left(\text { W,2): }: \diamond_{\operatorname{Tim}} \text { rich }(\text { bob: person })\right.}}
$$

First, the formula $(W, 0): \diamond_{\text {Sit }} \diamond_{\text {Tim }} F$ with three modal operators is annotated by the initial label $(W, 0)$. Second, $($ Sit, 1$): \diamond_{\text {Tim }} F$ is derived by deleting $\diamond_{\text {Sit }}$ where $\diamond_{\operatorname{Tim}} F$ is true in a situation world and the nested level is 1 . Third, $(W, 2): \diamond_{\text {Tim }} F$ is inferred by deleting $\diamond_{\text {where }} \diamond_{\text {Tim }} F$ is true in a world and the nested level is 2. Finally, (Tim, 3): $F$ is derived by deleting $\diamond_{\text {Tim }}$ where $F$ is true in a time world and the nested level is 3 . It is important that the pair 
of two labels (Tim,3) keeps the information that the current world is a time and the three modal operators are nested.

A ground term $t$ is of $(i, n)$ if the annotated term $t^{(i, n)}$ occurs in an ancestor. Let $i \in\left\{W, T_{1}, \ldots, T_{m}\right\}, j \in\left\{T_{1}, \ldots, T_{m}\right\}$, let $t$ be any ground term with $(i, n)$ , and let comma be the union of sets (i.e., $\Psi_{1}, \Psi_{2}=\Psi_{1} \cup \Psi_{2}, A, \Psi=\{A\} \cup \Psi$, and $A, B=\{A\} \cup\{B\}$ ). Each rule cannot be applied to a formula set if its conclusion has already been included in the formula set.

\section{Conjunction and disjunction rules}

$$
\frac{(i, n): A \wedge B, \Psi}{(i, n): A^{(i, n)}, B^{(i, n)}, \Psi}(\alpha) \quad \frac{(i, n): A \vee B, \Psi}{(i, n): A^{(i, n)}, \Psi \quad(i, n): B^{(i, n)}, \Psi}(\beta)
$$

The conjunction and disjunction rules are based on the standard tableau rules. It can be seen that if a formula is decomposed into atomic formulas, the ground terms in the atomic formulas are labeled with the natural number $n$, as $A^{(i, n)}$. This indicates that the included terms exist in world $n$, and they are referred to as terms with $(i, n)$. For example, the labeled formula $($ Tim, 3$): p\left(c^{(T i m, 3)}\right)$ indicates that the constant $c$ is an existing individual of the time world denoted by $($ Tim, 3$)$ and $p(c)$ is true in the world.

In $\alpha$-rule and $\beta$-rule, the decomposed formulas $A$ and $B$ are annotated with $(i, n)$ (such as $A^{(i, n)}$ and $B^{(i, n)}$ ) since they may be atomic formulas. For example, if $p(t) \wedge F$ is decomposed to $p(t)$ and $F$ by $\alpha$-rule, then we obtain the annotated atomic formula $p\left(t^{(i, n)}\right)$.

\section{Existential predicate rule}

$$
\frac{(i, n): \neg E(t), \Psi}{(i, n): \perp, \neg E(t), \Psi}(E)
$$

This rule is introduced to cover the existential predicate. In $E$-rule, if the term $t$ is labled by $(i, n)$, it derives a contradiction because $t$ must exist in world $n$.

In the modal operator rules, $* \Psi$ denotes $\{* F \mid F \in \Psi\}$ for $* \in\left\{\boldsymbol{\square}, \square_{1}, \ldots, \square_{m}\right\}$ (possibly $* \Psi=\emptyset$ ). Let $\mathcal{T}_{0}$ be the set of ground terms. The translation function $\mathcal{E}$ is defined by $\mathcal{E}\left(p\left(t_{1}, \ldots, t_{n}\right)\right)=\emptyset$ if $\left\{t_{1}, \ldots, t_{n}\right\} \nsubseteq \mathcal{T}_{0}$, otherwise $\mathcal{E}\left(p\left(t_{1}, \ldots\right.\right.$, $\left.\left.t_{n}\right)\right)=E\left(t_{1}\right) \wedge \cdots \wedge E\left(t_{n}\right)$. For any formula $F$, the function $\mathcal{E}$ can be expanded as follows:

(i) $\mathcal{E}(* F)=\mathcal{E}(F)$ for every $* \in\left\{\neg, \forall x_{s}, \exists x_{s}\right\}$,

(ii) $\mathcal{E}(* F)=\emptyset$ for every $* \in\left\{\square_{1}, \ldots, \square_{m}, \diamond_{1}, \ldots, \diamond_{m}, \mathbf{\square}, \diamond\right\}$,

(iii) $\mathcal{E}\left(F_{1} \wedge F_{2}\right)=\mathcal{E}\left(F_{1}\right) \wedge \mathcal{E}\left(F_{2}\right)$, and

(iv) $\mathcal{E}\left(F_{1} \vee F_{2}\right)=\mathcal{E}\left(F_{1}\right) \vee \mathcal{E}\left(F_{2}\right)$. 
Moreover, we define $\Psi \vee \neg \mathcal{E}(\Psi)=\{F \vee \neg \mathcal{E}(F) \mid F \in \Psi\}$. Moreover, we define $A \wedge \mathcal{E}(A)=A$ and $A \vee \neg \mathcal{E}(A)=A$ if $\mathcal{E}(A)=\emptyset$. Let $\Psi$ be a set of closed formulas. We denote $\Psi \wedge \mathcal{E}(\Psi)$ as $\{F \wedge \mathcal{E}(F) \mid F \in \Psi\}$ and $\Psi \vee \neg \mathcal{E}(\Psi)$ as $\{F \vee \neg \mathcal{E}(F) \mid$ $F \in \Psi\}$.

\section{Many modal operator rules}

$$
\begin{aligned}
& \frac{(j, n): \square_{j} A, \Psi}{(j, n): A \vee \neg \mathcal{E}(A), \square_{j} A, \Psi}\left(\nu_{j}\right) \quad \frac{(i, n): \diamond_{j} A, \square_{j} \Psi \cup \square \Psi, \Psi^{\prime}}{(j, n+1): A \wedge \mathcal{E}(A), \Psi \vee \neg \mathcal{E}(\Psi), \square \Psi}\left(\pi_{i \rightarrow j}\right) \\
& \frac{(j, n): \diamond_{j} A, \square_{j} \Psi \cup \boldsymbol{\square} \Psi, \Psi^{\prime}}{(j, n+1): A \wedge \mathcal{E}(A), \Psi \vee \neg \mathcal{E}(\Psi), \square_{j} \Psi \cup \mathbf{\square} \Psi}\left(\pi_{j}\right) \\
& \frac{(i, n): \diamond A, \mathbf{\square} \Psi, \Psi^{\prime}}{(W, n+1): A \wedge \mathcal{E}(A), \Psi \vee \neg \mathcal{E}(\Psi), \mathbf{\square} \Psi}\left(\pi_{W}\right) \\
& \frac{(i, n): \mathbf{\square} A, \Psi}{(i, n): A \vee \neg \mathcal{E}(A), \square_{1} A, \ldots, \square_{m} A, \mathbf{\square} A, \Psi}(\square) \frac{(i, n): \diamond_{j} A, \Psi}{(i, n): \diamond A, \diamond_{j} A, \Psi}(\diamond \diamond) \\
& \frac{(i, n): \diamond_{j} A, \Psi}{(i, n): A \wedge \mathcal{E}(A), \diamond \diamond_{j} A, \Psi}\left(\diamond \diamond^{+}\right)
\end{aligned}
$$

The $\nu_{j}$-rule derives the disjunction of the formula $A$ and the negation $\neg \mathcal{E}(A)$ of the existential predicate formula. In other words, the disjunction implies that the formula $A$ is true or some ground terms in $A$ do not exist.

The $\pi_{j}$-rule, $\pi_{i \rightarrow j}$-rule, and $\pi_{W}$-rule derive the conjunction of the formula $A$ and the existence $\mathcal{E}(A)$ of the ground terms in $A$ and these increase the natural number $n$ (by the annotation $(j, n+1)$ in the conclusion), since the modal operators $\diamond_{j}$ and imply that there exists a world $n+1$ accessible from $n$. In $\pi_{i \rightarrow j}$-rule, $i \neq j$, in $\pi_{j}$-rule and $\pi_{i \rightarrow j}$-rule, $\Psi^{\prime}$ is a set of closed formulas without the forms $F$ and $\square_{j} F$, and in $\pi_{W}$-rule, $\Psi^{\prime}$ is a set of closed formulas without the form $\square$. The $\pi_{j}$-rule does not modify the type of worlds but the $\pi_{i \rightarrow j}$ rule modifies the type of worlds $i$ into $j$ (by replacing the annotation $(i, n)$ with the annotation $(j, n+1))$. Further, when the $\pi_{W}$-rule is applied to a black diamond formula, the type of worlds is labeled as $W$ (by the annotation $(W, n+1)$ in the conclusion), denoting any type of worlds. Additionally, $\square \square$-rule and $\diamond \downarrow$ rule are introduced by supporting the fact that the possible worlds of time and situation are a subset of the set of worlds. If $\diamond_{j}$ is a knowledge operator, the $\diamond{ }^{+}$-rule is applied instead of the $\diamond$-rule.

The following sorted quantifier rules and sort predicate rules are applied to 
the quantifiers of typed variables and anti-rigid sorted variables.

\section{Sorted quantifier rules}

$$
\begin{aligned}
& \frac{(i, n): \forall x_{\tau} A, \Psi}{(i, n): A\left[x_{\tau} / t\right]^{(i, n)}, \forall x_{\tau} A, \Psi}\left(\gamma_{\tau}\right) \quad \frac{(i, n): p_{s^{\prime}}\left(t^{(i, n)}\right), \forall x_{s} A, \Psi}{(i, n): p_{s^{\prime}}\left(t^{(i, n)}\right), A\left[x_{s} / t\right]^{(i, n)}, \forall x_{s} A, \Psi}\left(\gamma_{s}\right) \\
& \frac{(i, n): \exists x_{\tau} A, \Psi}{(i, n): E\left(c_{\tau}^{(i, n)}\right), A\left[x_{\tau} / c_{\tau}\right]^{(i, n)}, \exists x_{\tau} A, \Psi}\left(\delta_{\tau}\right) \\
& \frac{(i, n): \exists x_{\sigma} A, \Psi}{(i, n): p_{\sigma}\left(c_{\tau}^{(i, n)}\right), A\left[x_{\sigma} / c_{\tau}\right]^{(i, n)}, \exists x_{\sigma} A, \Psi}\left(\delta_{\sigma}\right)
\end{aligned}
$$

In $\gamma_{\tau}$-rule, $\operatorname{sort}(t) \leq \tau$, in $\gamma_{s}$-rule, $s^{\prime} \leq s$ and $p_{s^{\prime}}$ is an anti-rigid sort predicate, in $\delta_{\tau}$-rule, $c_{\tau}$ is a constant not in $\left\{\exists x_{\tau} A\right\} \cup \Psi$, and in $\delta_{\sigma}$-rule, $c_{\tau}$ is a constant not in $\left\{\exists x_{\sigma} A\right\} \cup \Psi$ where $p_{\sigma}: \top \in \Omega$. Then, $\gamma_{\tau^{-}}$and $\delta_{\tau^{-}}$-rules are used for typed variables, $\delta_{s}$-rule is used for anti-rigid sorted variables, and the $\gamma_{s}$-rule is used for both typed and anti-rigid sorted variables.

The sort predicate rules derive the type and sort predicate formulas from a subsort relation. If the type of a term $t$ is a subtype of $\tau$, then this type predicate formula is true. Moreover, if $s$ is a subsort of $s^{\prime}$, then the sort predicate formula $p_{s}$ derives the sort predicate formula $p_{s}^{\prime}$.

\section{Sort predicate rules}

$$
\frac{(i, n): p_{s}\left(t^{(i, n)}\right), \Psi}{(i, n): p_{s^{\prime}}\left(t^{(i, n)}\right), p_{s}\left(t^{(i, n)}\right), \Psi}(<)
$$

where $s<s^{\prime}$.

\section{Type predicate rules}

$$
\frac{(i, n): \Psi}{(i, n): p_{\tau}\left(t^{(i, n)}\right), \Psi}\left(p_{\tau}\right) \quad \frac{(i, n): p_{\tau}\left(t^{(i, n)}\right), \Psi}{(i, n): \mathbf{\square}_{\tau}\left(t^{(i, n)}\right), \Psi}\left(\boldsymbol{\square}_{\tau}\right)
$$

where $\operatorname{sort}(t) \leq \tau$.

\section{Anti-rigid sort predicate rules}

$$
\frac{(i, n): p_{\sigma}\left(t^{(i, n)}\right), \Psi}{(i, n): \checkmark \neg p_{\sigma}\left(t^{(i, n)}\right), \Psi}\left(\diamond p_{\sigma}\right)
$$

A tableau rule is called static if it does not change the level $(i, n)$ (i.e., $(i, n): \Psi$ is expanded to $(i, n): \Psi^{\prime}$ by an application of the rule), it is called 
dynamic otherwise (e.g., $\pi_{j}$-rule, $\pi_{i \rightarrow j}$-rule, and $\pi_{W}$-rule are dynamic). The set of closed nodes in a tableau for $(i, n): \Psi$ is defined as follows:

(i) if a node contains two complementary literals $\left(\neg A\right.$ and $\left.A^{(i, n)}\right)$ or the clash symbol $\perp$, then it is closed, and

(ii) if all the children of a node are closed, then it is closed.

A tableau is closed if the root is closed.

\section{Completeness}

This section proves the completeness of our proposed tableau calculus by combining the techniques in order-sorted logic $[1,12,11]$ and in quantified modal logic [2].

Lemma 1 Let $M=\left(W, \vec{w}_{0},\left\{R_{1}, \ldots, R_{m}\right\}, R, U, I\right)$ be a sorted $\Sigma$-structure with existential rigidity and $A$ be a closed formula. For every $\vec{w} \in W$, the following statements hold:

1. $A \in N e x_{\vec{w}}$ if and only if $\vec{w} \mid \neq \mathcal{E}(A)$ and $\mathcal{E}(A) \neq \emptyset$.

2. If $A$ does not contain any ground term, then $A \notin N e x_{\vec{w}}$ and $\mathcal{E}(A)=\emptyset$.

Proof. By definition, it is trivial.

Lemma 2 Let $M=\left(W, \vec{w}_{0},\left\{R_{1}, \ldots, R_{m}\right\}, R, U, I\right)$ be a sorted $\Sigma$-structure with existential rigidity, let $\vec{w} \in W$, and let $t \in \mathcal{T}_{s, 0}$. If $\llbracket t \rrbracket_{\vec{w}}=d$, then $\vec{w}=$ $A\left[x_{s} / \bar{d}\right] \Leftrightarrow \vec{w}=A\left[x_{s} / t\right]$.

Proof. Similar to the proof of the counterpart in [2].

The following theorem shows the soundness of the labeled tableau calculus.

Theorem 1 If there exists a closed tableau for $\Psi$, then $\Psi$ is $\Sigma$-unsatisfiable.

Proof. Suppose that $\Psi$ is $\Sigma$-satisfiable. Then, there exists a sorted $\Sigma$-structure with existential rigidity $M=\left(W, \vec{w}_{0},\left\{R_{1}, \ldots, R_{m}\right\}, R, U, I\right)$ such that $\vec{w}_{0}=\Psi$. We will prove that there is no closed tableau for $\Psi$. Let $T$ be any tableau for $\Psi$. In order to show it, we construct a sub-tableau $T^{\prime}$ of $T$ such that the root $\Psi_{0}=S^{(W, 0)}$, each non-leaf node $\Psi_{k}$ has only one child $\Psi_{k+1}$, and every node is $\Sigma$-satisfiable (which implies that every node is not closed because every closed node is $\Sigma$-unsatisfiable). We show the satisfiability of each node $\Psi_{k}$ by induction on the depth $k$ of the tableau $T$. 
Base case: $k=0$. By the assumption, $M$ satisfies all the formulas in $\Psi$. So, $\vec{w}_{0}=\Psi^{+}$where $\Psi^{+}$is the non-annotated set of $S^{(W, 0)}$ in the initial tableau for $\Psi$.

Induction step: $k>0$.

$\left(\nu_{j}\right.$-rule) Let us assume $\vec{w} \models\left\{\square_{j} A\right\} \cup \Psi^{\prime}$ where $\vec{w} \in W$. Then, for all $\vec{w}^{\prime} \in W_{j}$ with $\left\langle\vec{w}, \vec{w}^{\prime}\right\rangle \in R_{j}, \vec{w}^{\prime} \mid=A$ or $A \in N e x_{\vec{w}^{\prime}}$. Since $R_{j}$ is reflexive, $\langle\vec{w}, \vec{w}\rangle \in R_{j}$, and so $\vec{w} \mid=A$ or $A \in N e x_{\vec{w}}$. This derives $\vec{w}=A \vee \neg \mathcal{E}(A)$ by Lemma 1 (1).

( $\pi_{j}$-rule) Let us assume $\vec{w} \models\left\{\diamond_{j} A\right\} \cup \square_{j} \Psi^{\prime} \cup \Psi^{\prime} \cup \Psi^{\prime \prime}$ where $\vec{w} \in W$. For some $\vec{w}_{a} \in W_{j}$ with $\left\langle\vec{w}, \vec{w}_{a}\right\rangle \in R_{j}, \vec{w}_{a}=A$ and $A \notin N e x_{\vec{w}_{a}}$, and thus $\vec{w}_{a}=A \wedge \mathcal{E}(A)$. Let $F \in\left\{F \mid \square_{j} F \in \square_{j} \Psi^{\prime} \cup \square \Psi^{\prime}\right\}$. By the assumption, for all $\vec{w}^{\prime} \in W_{j}$ with $\left\langle\vec{w}, \vec{w}^{\prime}\right\rangle \in R_{j}, \vec{w}^{\prime} \models F$ or $F \in N e x_{\vec{w}^{\prime}}$. So, $\vec{w}_{a} \models F \vee \neg \mathcal{E}(F)$ by Lemma 1 (1). Moreover, since $R_{j}$ is transitive, if $\left\langle\vec{w}, \vec{w}_{a}\right\rangle$ and $\left\langle\vec{w}_{a}, \vec{w}_{a}^{\prime}\right\rangle$ in $R_{j}$, then $\left\langle\vec{w}, \vec{w}_{a}^{\prime}\right\rangle$ in $R_{j}$. This implies that for all $\vec{w}^{\prime \prime} \in W_{j}$ with $\left\langle\vec{w}_{a}, \vec{w}^{\prime \prime}\right\rangle \in R_{j}, \vec{w}^{\prime \prime} \mid=F$ or $F \in N e x_{\vec{w}^{\prime \prime}}$ (i.e., $\vec{w}_{a} \models \square_{j} F$ ). Let $F \in\left\{F \mid \boldsymbol{\square} F \in \square_{j} \Psi^{\prime} \cup \mathbf{\square} \Psi^{\prime}\right\}$. By the assumption, for all $\vec{w}^{\prime} \in W$ with $\left\langle\vec{w}, \vec{w}^{\prime}\right\rangle \in R, \vec{w}^{\prime}=F$ or $F \in N e x_{\vec{w}^{\prime}}$. Since $\left\langle\vec{w}, \vec{w}_{a}\right\rangle \in R_{j}(\subseteq R)$, $\vec{w}_{a}=F \vee \neg \mathcal{E}(F)$ by Lemma 1 (1). Since $R$ is transitive, if $\left\langle\vec{w}, \vec{w}_{a}\right\rangle$ and $\left\langle\vec{w}_{a}, \vec{w}_{a}^{\prime}\right\rangle$ in $R$, then $\left\langle\vec{w}, \vec{w}_{a}^{\prime}\right\rangle$ in $R$. Hence, for all $\vec{w}^{\prime \prime} \in W$ with $\left\langle\vec{w}_{a}, \vec{w}^{\prime \prime}\right\rangle \in R, \vec{w}^{\prime \prime} \models F$ or $F \in N_{e x} \vec{w}_{\vec{w}^{\prime \prime}}$ (i.e., $\vec{w}_{a} \models \mathbf{\square} F$ ).

$\left(\pi_{i \rightarrow j}\right.$-rule) Let us assume $\vec{w}=\left\{\diamond_{j} A\right\} \cup \square_{j} \Psi^{\prime} \cup \square \Psi^{\prime} \cup \Psi^{\prime \prime}$ with $i \neq j$ where $\vec{w} \in W$ if $i=W, w \in W_{i}$ otherwise. For some $\vec{w}_{a} \in W_{j}$ with $\left\langle\vec{w}, \vec{w}_{a}\right\rangle \in R_{j}$, $\vec{w}_{a} \models A$ and $A \notin N e x_{\vec{w}_{a}}$. So, $\vec{w}_{a} \models A \wedge \mathcal{E}(A)$. Let $F \in\left\{F \mid \square_{j} F \in \square_{j} \Psi^{\prime} \cup \square \Psi^{\prime}\right\}$. By the assumption, for all $\vec{w}^{\prime} \in W_{j}$ with $\left\langle\vec{w}, \vec{w}^{\prime}\right\rangle \in R_{j}, \vec{w}^{\prime}=F$ or $F \in N e x_{\vec{w}^{\prime}}$. So, $\vec{w}_{a}=F \vee \neg \mathcal{E}(F)$ by Lemma $1(1)$. Let $F \in\left\{F \mid \boldsymbol{\nabla} F \in \square_{j} \Psi^{\prime} \cup \boldsymbol{\square} \Psi^{\prime}\right\}$. By the assumption, for all $\vec{w}^{\prime} \in W$ with $\left\langle\vec{w}, \vec{w}^{\prime}\right\rangle \in R, \vec{w}^{\prime} \models F$ or $F \in N e x_{\vec{w}^{\prime}}$. Because $\left\langle\vec{w}, \vec{w}_{a}\right\rangle \in R_{j}(\subseteq R), \vec{w}_{a} \models F \vee \neg \mathcal{E}(F)$ by Lemma 1 (1). Moreover, since $R$ is transitive, if $\left\langle\vec{w}, \vec{w}_{a}\right\rangle$ and $\left\langle\vec{w}_{a}, \vec{w}_{a}^{\prime}\right\rangle$ in $R$, then $\left\langle\vec{w}, \vec{w}_{a}^{\prime}\right\rangle$ in $R$. This implies that for all $\vec{w}^{\prime \prime} \in W$ with $\left\langle\vec{w}_{a}, \vec{w}^{\prime \prime}\right\rangle \in R, \vec{w}^{\prime \prime}=F$ or $F \in N e x_{\vec{w}^{\prime \prime}}$ (i.e., $\vec{w}_{a}=\mathbf{\square} F$ ).

$\left(\pi_{W}\right.$-rule) Let us assume $\vec{w} \models\{\forall\} \cup \square \Psi^{\prime} \cup \Psi^{\prime \prime}$. For some $\vec{w}_{a} \in W$ with $\left\langle\vec{w}, \vec{w}_{a}\right\rangle \in R, \vec{w}_{a} \models A$ and $A \notin N e x_{\vec{w}_{a}}$. Hence, $\vec{w}_{a} \models A \wedge \mathcal{E}(A)$. Let $F \in\{F \mid$ $\left.\mathbf{\square} F \in \Psi^{\prime}\right\}$. By the assumption, for all $\vec{w}^{\prime} \in W$ with $\left\langle\vec{w}, \vec{w}^{\prime}\right\rangle \in R, \vec{w}^{\prime} \mid=F$ or $F \in N e x_{\vec{w}^{\prime}}$. So, $\vec{w}_{a}=F \vee \neg \mathcal{E}(F)$ by Lemma 1 (1). Moreover, since $R$ is transitive, if $\left\langle\vec{w}, \vec{w}_{a}\right\rangle$ and $\left\langle\vec{w}_{a}, \vec{w}_{a}^{\prime}\right\rangle$ in $R$, then $\left\langle\vec{w}, \vec{w}_{a}^{\prime}\right\rangle$ in $R$. Therefore, for all $\vec{w}^{\prime \prime} \in W$ with $\left\langle\vec{w}_{a}, \vec{w}^{\prime \prime}\right\rangle \in R, \vec{w}^{\prime \prime} \models F$ or $F \in N e x_{\vec{w}^{\prime \prime}}$ (i.e., $\vec{w}_{a}=\mathbf{\square} F$ ).

(品-rule) Let us assume $\vec{w}=\{\boldsymbol{\square} A\} \cup \Psi^{\prime}$. For all $\vec{w}^{\prime} \in W$ with $\left\langle\vec{w}, \vec{w}^{\prime}\right\rangle \in R$, $\vec{w}^{\prime} \models A$ or $A \in N e x_{\vec{w}^{\prime}}$. $R_{i}$ is a subset of $R$. So, for all $\vec{w}^{\prime} \in W$ with $\left\langle\vec{w}, \vec{w}^{\prime}\right\rangle \in R_{i}$, $\vec{w}^{\prime}=A$ or $A \in N_{e x} x_{\vec{w}^{\prime}}$. Hence, $\vec{w}=\square_{\text {Tim }} A$ and $\vec{w}=\square_{\text {Sit }} A$. Since $R$ is reflexive, $\langle\vec{w}, \vec{w}\rangle \in R$, and by Lemma $1(1), \vec{w}=A \vee \neg \mathcal{E}(A)$.

$\left(\diamond \diamond\right.$-rule) Let us assume $\vec{w}=\left\{\diamond_{j} A\right\} \cup \Psi^{\prime}$ where $\diamond_{j}$ is a knowledge operator. For some $\vec{w}_{a} \in W_{j}$ with $\left\langle\vec{w}, \vec{w}_{a}\right\rangle \in R_{j}, \vec{w}_{a}=A$ and $A \notin N e x_{\vec{w}_{a}}$ (i.e., $\vec{w}_{a}=$ $A \wedge \mathcal{E}(A))$. Due to $R_{j} \subseteq R$ and $W_{j} \subseteq W,\left\langle\vec{w}, \vec{w}_{a}\right\rangle \in R$ and $\vec{w}_{a} \in W$. From this $\vec{w} \models A$ follows. 
$\left(\diamond \diamond^{+}\right.$-rule) Let us assume $\vec{w}=\left\{\diamond_{j} A\right\} \cup \Psi^{\prime}$. By the soundness of $\square$-rule, for some $\vec{w}_{a} \in W_{j}$ with $\left\langle\vec{w}, \vec{w}_{a}\right\rangle \in R_{j}, \vec{w}_{a} \models A \wedge \mathcal{E}(A)$ and $\vec{w} \models A$ follows. Because $R_{i}$ is symmetric, $\left\langle\vec{w}_{a}, \vec{w}\right\rangle \in R_{j}, \vec{w}=A \wedge \mathcal{E}(A)$.

$\left(\gamma_{\tau}\right.$-rule) Let $\vec{w}=\left\{\forall x_{\tau} A\right\} \cup \Psi^{\prime}$. For all $d \in I_{\vec{w}}(\tau), \vec{w} \models A\left[x_{\tau} / \bar{d}\right]$. Since $\operatorname{sort}(t) \leq \tau$ and $t$ is a ground term with $(i, n), \llbracket t \rrbracket_{\vec{w}}=d^{\prime} \in U_{\vec{w}} \cap I_{\vec{w}}(\tau)\left(\subseteq I_{\vec{w}}(\tau)\right)$. So, $\vec{w}=A\left[x_{\tau} / \bar{d}^{\prime}\right]$. This yields $\vec{w}=A\left[x_{\tau} / t\right]$.

$\left(\gamma_{s^{-}}\right.$-rule) Let $\vec{w}=\left\{p_{s^{\prime}}(t), \forall x_{s} A\right\} \cup \Psi^{\prime}$ where $s^{\prime} \leq s$. Then, $\llbracket t \rrbracket_{\vec{w}} \in I_{\vec{w}}\left(p_{s^{\prime}}\right)$, and for all $d \in I_{\vec{w}}(s), \vec{w}=A\left[x_{s} / \bar{d}\right]$. If $s$ is extensible, then $p_{s^{\prime}}$ is an anti-rigid sort predicate, $I_{\vec{w}}\left(p_{s^{\prime}}\right)=I_{\vec{w}}\left(s^{\prime}\right)\left(\subseteq I_{\vec{w}}(s)\right)$. If $s$ is inextensible, then $\left(I_{\vec{w}}\left(p_{s^{\prime}}\right) \subseteq\right)$ $I_{\vec{w}}\left(p_{s}\right)=I_{\vec{w}}(s)$. Thus, $\llbracket t \rrbracket_{\vec{w}}=d^{\prime} \in I_{\vec{w}}(s)$, and $\vec{w} \models A\left[x_{s} / \bar{d}^{\prime}\right]$. It follows that $w \models A\left[x_{s} / t\right]$.

$\left(\delta_{\tau}\right.$-rule) Let $\vec{w} \models\left\{\exists x_{\tau} A\right\} \cup \Psi^{\prime}$. For some $d \in I_{\vec{w}}(\tau), \vec{w} \models A\left[x_{\tau} / \bar{d}\right]$. Let us extend $M$ to $M^{\prime}$ by setting $I_{\vec{w}}(c)=d\left(\in I_{\vec{w}}(\tau) \subseteq U_{\vec{w}}\right)$ where $c_{\tau}$ is not in $\left\{\exists x_{\tau} A\right\} \cup \Psi^{\prime}$ and $c: \rightarrow \tau \in \Omega$. Therefore, $w=E\left(c_{\tau}\right)$ and $w \models A\left[x_{\tau} / c_{\tau}\right]$ in $M^{\prime}$.

$\left(\delta_{\sigma}\right.$-rule) Let $\vec{w} \models\left\{\exists x_{\sigma} A\right\} \cup \Psi^{\prime}$ where $p_{\sigma}: \tau \in \Omega$. For some $d \in I_{\vec{w}}(\sigma)$, $\vec{w}=A\left[x_{\sigma} / \bar{d}\right]$. Let us extend $M$ to $M^{\prime}$ by setting $I_{\vec{w}}(c)=d\left(\in I_{\vec{w}}(\sigma)\right)$ where $c_{\tau}$ is not in $\left\{\exists x_{\sigma} A\right\} \cup \Psi^{\prime}$ and $c: \rightarrow \tau \in \Omega$. Thus, $\vec{w}=A\left[x_{\sigma} / c_{\tau}\right]$ in $M^{\prime}$. Because of $I_{\vec{w}}(\sigma)=I_{\vec{w}}\left(p_{\sigma}\right), w=p_{\sigma}\left(c_{\tau}\right)$ in $M^{\prime}$.

(<-rule) Let $\vec{w}=\left\{p_{s}(t)\right\} \cup \Psi^{\prime}$. If $s \leq s^{\prime}$, then by definition, $I_{\vec{w}}\left(p_{s}\right) \subseteq I_{\vec{w}}\left(p_{s^{\prime}}\right)$. Hence, $\vec{w}=p_{s^{\prime}}(t)$.

(E-rule) Let us assume $\vec{w}=\{\neg E(t)\} \cup \Psi^{\prime}$ where $t$ is a ground term with $(i, n)$. Then, $t^{(i, n)}$ occurs in a positive atomic formula (i.e., $\Psi^{\prime}$ contains (i) an atomic formula $p\left(t_{1}^{(i, n)}, \ldots, t_{l}^{(i, n)}\right)$ where $t=t_{u}$ for some $u \in\{1, \ldots, l\}$ or (ii) $\left.E\left(t^{(i, n)}\right)\right)$. For (i), $\left(\llbracket t_{1} \rrbracket_{\vec{w}}, \ldots, \llbracket t_{l} \rrbracket_{\vec{w}}\right) \in I_{\vec{w}}(p)\left(\subseteq U_{\vec{w}} \times \cdots \times U_{\vec{w}}\right)$, so that $\llbracket t \rrbracket_{\vec{w}} \in U_{\vec{w}}$. Thus, (i) and (ii) imply $\vec{w}=E(t)$. It is a contradiction.

( $\alpha$-rule), ( $\beta$-rule), and ( $I$-rule) For the cases, it is easy to show that each tableau rule preserves satisfiability.

( $p_{\tau}$-rule) Let $\vec{w} \models \Psi^{\prime}$, and let $t$ be a ground term such that $\operatorname{sor} t(t) \leq \tau$. Then, $\llbracket t \rrbracket_{\vec{w}} \in U_{\vec{w}} \cap I_{\vec{w}}(\tau)$, and by the definition of sorted $\Sigma$-structures, $\llbracket t \rrbracket_{\vec{w}} \in I_{\vec{w}}(\tau)$. Since $I_{\vec{w}}(\tau) \subseteq I_{\vec{w}}\left(p_{\tau}\right)$, we obtain $\vec{w}=p_{\tau}(t)$.

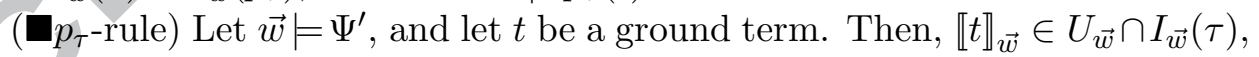
and by the definition of existential rigidity, for any world $\vec{w}^{\prime}$ with $\left\langle\vec{w}, \vec{w}^{\prime}\right\rangle \in R$, $\llbracket t \rrbracket_{\vec{w}} \notin U_{\vec{w}^{\prime}}(\tau)$ or $\llbracket t \rrbracket_{\vec{w}} \in I_{\vec{w}^{\prime}}(\tau)$. Since $I_{\vec{w}^{\prime}}(\tau) \subseteq I_{\vec{w}^{\prime}}\left(p_{\tau}\right)$, we obtain $\vec{w}=\mathbf{\square}_{p_{\tau}}(t)$.

$\left(p_{\sigma}\right.$-rule) Let $\vec{w} \models \Psi^{\prime}$, and let $t$ be a ground term. Then, $\llbracket t \rrbracket_{\vec{w}} \in U_{\vec{w}} \cap I_{\vec{w}}(\sigma)$, and by the definition of existential rigidity, there exists $\vec{w}_{j} \in W$ with $\left\langle\vec{w}, \vec{w}_{j}\right\rangle \in$ $R$ such that $\llbracket t \rrbracket_{\vec{w}} \notin I_{\vec{w}_{j}}(\sigma)$ with $\llbracket t \rrbracket_{\vec{w}} \in U_{\vec{w}_{j}}$. Therefore, $\vec{w}=\neg p_{\tau}(t)$.

Therefore, since every node $\Psi_{k}$ in the sub-tableau $T^{\prime}$ is $\Sigma$-satisfiable, it is not closed.

In order to prove the completeness of the labeled tableau calculus, we need to define saturated sets of formulas and a canonical interpretation of a formula set. Let $(i, n): \Psi$ be a labeled set of closed formulas. We denote the set of 
ground terms of sort $s$ with $(i, n)$ in $\Psi$ by $\mathcal{T}_{s, 0}((i, n): \Psi)=\left\{t \in \mathcal{T}_{0} \cap \mathcal{T}_{s} \mid\right.$ $t^{(i, n)}$ occurs in $\left.\Psi\right\}$. We represent the set of ground terms of a sort predicate $p_{s}$ with $(i, n) . \quad \mathcal{T}_{p_{s}, 0}((i, n): \Psi)=\bigcup_{s^{\prime} \leq s}\left\{t \in \mathcal{T}_{0} \mid p_{s^{\prime}}\left(t^{(i, n)}\right) \in \Psi\right\}$. The set $\mathcal{T}_{p_{s}, 0}((i, n): \Psi)$ consists of the ground terms $t$ in atomic sort predicate formulas $p_{s^{\prime}}\left(t^{(i, n)}\right)$ for all the sorts $s^{\prime}$ with $s^{\prime} \leq s^{1}$.

Lemma 3 Let $\Sigma=\left(T, S_{A}, \leq, \Omega\right)$ be a sorted signature and $(i, n): \Psi$ be a labeled set of closed formulas. If $s \leq s^{\prime}$, then $\mathcal{T}_{s, 0}((i, n): \Psi) \subseteq \mathcal{T}_{s^{\prime}, 0}((i, n): \Psi)$ and $\mathcal{T}_{p_{s}, 0}((i, n): \Psi) \subseteq \mathcal{T}_{p_{s^{\prime}}, 0}((i, n): \Psi)$.

Proof. By definition, it is easy to show.

A set $\Psi$ of closed formulas is consistent if its non-annotated set does not contain any pair of complementary literals (i.e., $\neg A$ and $A$ ) or the clash symbol $\perp$. For example, if $\left\{\neg p(t), p\left(t^{(i, n)}\right)\right\} \subseteq \Psi$, then it is not consistent.

Definition 10 A labeled set $(i, n): \Psi$ is saturated if $\Psi$ is consistent and the following rules are satisfied:

1. $p_{\tau}\left(t^{(i, n)}\right) \in \Psi$ for every ground term $t \in \mathcal{T}_{\tau, 0}((i, n): \Psi)$.

2. if $p_{s}\left(t^{(i, n)}\right) \in \Psi$ and $s \leq s^{\prime}$, then $p_{s^{\prime}}\left(t^{(i, n)}\right) \in \Psi$.

3. if $p_{\tau}\left(t^{(i, n)}\right) \in \Psi$, then $\mathbf{p}_{\tau}\left(t^{(i, n)}\right) \in \Psi$.

4. if $p_{\sigma}\left(t^{(i, n)}\right) \in \Psi$, then $\neg p_{\sigma}\left(t^{(i, n)}\right) \in \Psi$.

5. if $F_{1} \wedge F_{2} \in \Psi$, then $F_{1}^{(i, n)} \in \Psi$ and $F_{2}^{(i, n)} \in \Psi$.

6. if $F_{1} \vee F_{2} \in \Psi$, then either $F_{1}^{(i, n)} \in \Psi$ or $F_{2}^{(i, n)} \in \Psi$.

7. if $\left(\forall x_{s}\right) F \in \Psi$, then $F\left[x_{s} / t\right]^{(i, n)} \in \Psi$ for every ground term $t \in \mathcal{T}_{s, 0}((i, n): \Psi) \cup$ $\mathcal{T}_{p_{s}, 0}((i, n): \Psi)$.

8. if $\left(\exists x_{s}\right) F \in \Psi$, then $p_{s}\left(c_{\tau}^{(i, n)}\right), F\left[x_{s} / c_{\tau}\right]^{(i, n)} \in \Psi$ for some constant $c_{\tau}$ where $\tau=s$ if $s \in T, p_{s}: \tau \in \Omega$ otherwise.

9. if $\square_{i} F \in \Psi$, then $F \vee \neg \mathcal{E}(F) \in \Psi$.

10. if $\boldsymbol{\square} F \in \Psi$, then $F \vee \neg \mathcal{E}(F), \square_{1} F, \ldots, \square_{m} F \in \Psi$.

11. if $\diamond_{i} F \in \Psi$, then $\diamond F \in \Psi$.

12. if $\diamond_{i} F \in \Psi$ and $\diamond_{j}$ is a knowledge operator, then $A \wedge \mathcal{E}(A), \diamond F \in \Psi$.

\footnotetext{
${ }^{1}$ Every subsort relation is reflexive, antisymmetric, and transitive since it is a partial order.
} 
A labeled set $(i, n): \Psi$ is tab-consistent if for every finite subset $\Psi^{\prime}$ of $\Psi$ there is no closed tableau for $(i, n): \Psi^{\prime}$.

Lemma 4 If $(i, n): \Psi$ is tab-consistent, then there exists a superset $\Psi^{*}$ of $\Psi$ such that $(i, n): \Psi^{*}$ is saturated.

Proof. Let a labeled set $(i, n): \Psi$ be tab-consistent. We inductively construct a sequence $(i, n): \Psi_{0},(i, n): \Psi_{1}, \ldots,(i, n): \Psi_{k}, \ldots$ of nodes by applications of static tableau rules where $\Psi_{0}=\Psi$.

$(k>0)$ Let a static tableau rule of the form $\frac{(i, n): \Psi_{k}}{(i, n): \Psi_{k+1}}$ be applied. Since $(i, n): \Psi_{k}$ is tab-consistent, $(i, n): \Psi_{k+1}$ is tab-consistent. This is because every static rule does not delete any pair of complementary literals (i.e., $\neg A$ and $A$ ) or the clash symbol $\perp$. Let a static tableau rule of the form $\frac{(i, n): \Psi_{k}}{(i, n): \Psi^{\prime}(i, n): \Psi^{\prime \prime}}$ be applied. Since $(i, n): \Psi_{k}$ is tab-consistent, $(i, n): \Psi^{\prime}$ or $(i, n): \Psi^{\prime \prime}$ is tabconsistent. We define $\Psi_{k+1}=\Psi^{\prime}$ if $(i, n): \Psi^{\prime}$ is tab-consistent, $\Psi_{k+1}=\Psi^{\prime \prime}$ otherwise.

It remains to prove that there exists a superset $\Psi^{*}$ of $\Psi$ such that $(i, n): \Psi^{*}$ is saturated.

Let $\Psi^{*}=\bigcup_{k \in \mathbb{N}} \Psi_{k}$. Assume that the non-annotated set of $\Psi^{*}$ contains a pair of an atomic formula $A$ and its negation $\neg A$ or the clash $\perp$. For each set $\Psi_{k}$, let us denote the set of literals and $\perp$ occurring in $\Psi_{k}$ by $L\left(\Psi_{k}\right)$. Since every static rule does not delete them, $L\left(\Psi_{0}\right) \subseteq L\left(\Psi_{1}\right) \subseteq \cdots \subseteq L\left(\Psi_{k}\right) \subseteq \cdots$. By the assumption, there exists $m \in \mathrm{N}$ such that $\Psi_{m}$ is not consistent. This is contradictory to the fact that every node $(i, n): \Psi_{k}$ is tab-consistent. It follows that $\Psi^{*}$ is consistent.

We need to check that $\Psi^{*}$ satisfies Conditions (1) - (12) in Definition 10. Condition (1). By $p_{\tau}$-rule, this condition is satisfied. Condition (2). By $<$-rule, this condition is satisfied. Conditions (3) and (4). By $p_{\tau}$-rule and $p_{\sigma}$-rule, these conditions are satisfied. Conditions (5) and (6). By $\alpha$-rule and $\beta$-rule,

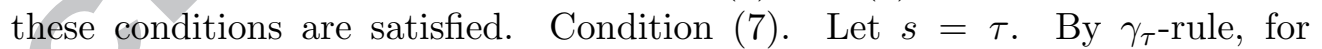
every $t \in \mathcal{T}_{\tau, 0}\left((i, n): \Psi^{*}\right), F\left[x_{\tau} / t\right]^{(i, n)} \in \Psi^{*}$. Let $t \in \mathcal{T}_{p_{\tau}, 0}\left((i, n): \Psi^{*}\right)$. Then, $F\left[x_{\tau} / t\right]^{(i, n)} \in \Psi^{*}$ by $\gamma_{s}$-rule. Let $s=\sigma . \mathcal{T}_{\sigma, 0}\left((i, n): \Psi^{*}\right)$ is the empty set since $\mathcal{T}_{0} \cap \mathcal{T}_{\sigma}=\emptyset$ (by Definition 2). For every $t \in \mathcal{T}_{p_{\sigma}, 0}\left((i, n): \Psi^{*}\right)$, there exists $\sigma^{\prime}$ such that $\sigma^{\prime} \leq \sigma$ and $p_{\sigma^{\prime}}\left(t^{(i, n)}\right) \in \Psi^{*}$. By $\gamma_{s^{-}}$rule, $F\left[x_{\sigma} / t\right]^{(i, n)} \in \Psi^{*}$. Condition (8). Let $s=\tau$. By $\delta_{\tau^{-r u l e}} E\left(c_{\tau}^{(i, n)}\right), F\left[x_{\tau} / c_{\tau}\right]^{(i, n)} \in \Psi^{*}$, and thus, by $p_{\tau}$-rule, $p_{\tau}\left(c_{\tau}^{(i, n)}\right) \in \Psi^{*}$. Let $s=\sigma$ with $p_{\sigma}: \tau \in \Omega$. By $\delta_{\sigma^{-r u l e}} p_{\sigma}\left(c_{\tau}^{(i, n)}\right), F\left[x_{\sigma} / c_{\tau}\right]^{(i, n)} \in$

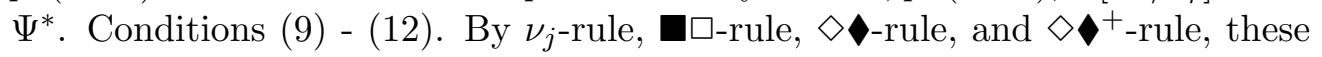
conditions are satisfied.

These yield the conclusion that $(i, n): \Psi^{*}$ is saturated.

The following lemma will be used to construct a canonical interpretation. 
Lemma 5 Let $(i, n): \Psi$ be tab-consistent and let $j \in\{1, \ldots, m\}$. The following statements hold:

1. if $\Psi=\diamond_{j} A, \square_{j} \Psi^{\prime} \cup \square \Psi^{\prime}, \Psi^{\prime \prime}(i=j)$, then $(j, n+1): A \wedge \mathcal{E}(A), \Psi^{\prime} \vee$ $\neg \mathcal{E}\left(\Psi^{\prime}\right),\left\langle\square_{j}, \mathbf{\square}\right\rangle \Psi^{\prime}$ is tab-consistent.

2. if $\Psi=\diamond_{j} A, \square_{j} \Psi^{\prime} \cup \mathbf{\square} \Psi^{\prime}, \Psi^{\prime \prime}(i \neq j)$, then $(j, n+1): A \wedge \mathcal{E}(A), \Psi^{\prime} \vee$ $\neg \mathcal{E}\left(\Psi^{\prime}\right), \boldsymbol{\square} \Psi^{\prime}$ is tab-consistent.

3. if $\Psi=\triangleleft A, \boldsymbol{\square} \Psi^{\prime}, \Psi^{\prime \prime}$, then $(W, n+1): A \wedge \mathcal{E}(A), \Psi^{\prime} \vee \neg \mathcal{E}\left(\Psi^{\prime}\right), \mathbf{\square} \Psi^{\prime}$ is tabconsistent.

Proof. (1) Let us suppose that $(j, n+1): A \wedge \mathcal{E}(A), \Psi^{\prime} \vee \neg \mathcal{E}\left(\Psi^{\prime}\right), \square_{j} \Psi^{\prime} \cup \square \Psi^{\prime}$ is not tab-consistent. Then, for some finite subset $\Psi_{l}$ of it, there exists a closed tableau for $(j, n+1): \Psi_{l}$. We can set $\Psi_{l}=\left(\Psi_{1} \wedge \mathcal{E}\left(\Psi_{1}\right)\right) \cup\left(\Psi_{2} \vee \neg \mathcal{E}\left(\Psi_{2}\right)\right) \cup \Psi_{3} \cup \Psi_{4}$ where $\Psi_{1} \subseteq\{A\}, \Psi_{2} \subseteq \Psi^{\prime}, \Psi_{3} \subseteq \square_{j} \Psi^{\prime}$, and $\Psi_{4} \subseteq \mathbf{\square} \Psi^{\prime}$. Let $\Psi_{l}^{\prime}=\{A \wedge \mathcal{E}(A)\} \cup$ $\left(\Psi_{2}^{\prime} \vee \neg \mathcal{E}\left(\Psi_{2}^{\prime}\right)\right) \cup \Psi_{3}^{\prime} \cup \Psi_{4}^{\prime}$ where $\Psi_{2}^{\prime}=\Psi_{2} \cup\left\{B \mid \square_{j} B \in \Psi_{3}\right\} \cup\left\{B \mid \mathbf{\square} B \in \Psi_{4}\right\}$, $\Psi_{3}^{\prime}=\Psi_{3} \cup \square_{j} \Psi_{2}$, and $\Psi_{4}^{\prime}=\Psi_{4} \cup \mathbf{\square} \Psi_{2}$. Because of $\Psi_{l} \subseteq \Psi_{l}^{\prime}$, there exists a closed tableau for $(j, n+1): \Psi_{l}^{\prime}$. Due to $\square_{j} \Psi_{2}^{\prime} \cup \square \Psi_{2}^{\prime}=\Psi_{3}^{\prime} \cup \Psi_{4}^{\prime}, \pi_{j}$-rule derives $(j, n+1): \Psi_{l}^{\prime}$ from $(i, n): \diamond_{j} A, \square_{j} \Psi_{2}^{\prime} \cup \Psi_{2}^{\prime}$. Since $\Psi_{2}^{\prime} \subseteq \Psi^{\prime},\left\{\diamond_{j} A\right\} \cup \square_{j} \Psi_{2}^{\prime} \cup \square \Psi_{2}^{\prime}$ is a finite subset of $\Psi$. Hence, $\Psi$ is not tab-consistent.

(2) Suppose that $(j, n+1): A \wedge \mathcal{E}(A), \Psi^{\prime} \vee \neg \mathcal{E}\left(\Psi^{\prime}\right), \mathbf{\square} \Psi^{\prime}$ is not tab-consistent. Then, for some finite subset $\Psi_{l}$ of it, there exists a closed tableau for $(j, n+$ 1): $\Psi_{l}$. We can set $\Psi_{l}=\left(\Psi_{1} \wedge \mathcal{E}\left(\Psi_{1}\right)\right) \cup\left(\Psi_{2} \vee \neg \mathcal{E}\left(\Psi_{2}\right)\right) \cup \Psi_{3}$ where $\Psi_{1} \subseteq\{A\}$, $\Psi_{2} \subseteq \Psi^{\prime}$, and $\Psi_{3} \subseteq \mathbf{\square} \Psi^{\prime}$. Let $\Psi_{l}^{\prime}=\{A \wedge \mathcal{E}(A)\} \cup\left(\Psi_{2}^{\prime} \vee \neg \mathcal{E}\left(\Psi_{2}^{\prime}\right)\right) \cup \Psi_{3}^{\prime}$ where $\Psi_{2}^{\prime}=\Psi_{2} \cup\left\{B \mid \boldsymbol{\nabla} B \in \Psi_{3}\right\}$ and $\Psi_{3}^{\prime}=\Psi_{3} \cup \mathbf{\square} \Psi_{2}$. Because of $\Psi_{l} \subseteq \Psi_{l}^{\prime}$, there exists a closed tableau for $(j, n+1): \Psi_{l}^{\prime}$. Due to $\Psi_{2}^{\prime}=\Psi_{3}^{\prime}, \pi_{i \rightarrow j}$-rule infers $(j, n+1): \Psi_{l}^{\prime}$ from $(i, n): \diamond_{j} A, \square_{j} \Psi_{2}^{\prime} \cup \boldsymbol{\nabla} \Psi_{2}^{\prime}$. Since $\Psi_{2}^{\prime} \subseteq \Psi^{\prime},\left\{\diamond_{j} A\right\} \cup \square_{j} \Psi_{2}^{\prime} \cup \boldsymbol{\square} \Psi_{2}^{\prime}$ is a finite subset of $\Psi$. Thus, $\Psi$ is not tab-consistent.

(3) Let us suppose that $(W, n+1): A \wedge \mathcal{E}(A), \Psi^{\prime} \vee \neg \mathcal{E}\left(\Psi^{\prime}\right), \boldsymbol{\square} \Psi^{\prime}$ is not tabconsistent. Then, for some finite subset $\Psi_{l}$ of it, there exists a closed tableau for $(W, n+1): \Psi_{l}$. We can set $\Psi_{l}=\left(\Psi_{1} \wedge \mathcal{E}\left(\Psi_{1}\right)\right) \cup\left(\Psi_{2} \vee \neg \mathcal{E}\left(\Psi_{2}\right)\right) \cup \Psi_{3}$ where $\Psi_{1} \subseteq\{A\}, \Psi_{2} \subseteq \Psi^{\prime}$, and $\Psi_{3} \subseteq \mathbf{\square} \Psi^{\prime}$. Moreover, let $\Psi_{l}^{\prime}=\{A \wedge \mathcal{E}(A)\} \cup\left(\Psi_{2}^{\prime} \vee\right.$ $\left.\neg \mathcal{E}\left(\Psi_{2}^{\prime}\right)\right) \cup \Psi_{3}^{\prime}$ where $\Psi_{2}^{\prime}=\Psi_{2} \cup\left\{B \mid \boldsymbol{\square} B \in \Psi_{3}\right\}$ and $\Psi_{3}^{\prime}=\Psi_{3} \cup \boldsymbol{\nabla} \Psi_{2}$. Because of $\Psi_{l} \subseteq \Psi_{l}^{\prime}$, there exists a closed tableau for $(W, n+1): \Psi_{l}^{\prime}$. Due to $\mathbf{\square} \Psi_{2}^{\prime}=\Psi_{3}^{\prime}, \pi_{W^{-}}$ rule derives $(W, n+1): \Psi_{l}^{\prime}$ from $(i, n): \checkmark A, \Psi_{2}^{\prime}$. Since $\Psi_{2}^{\prime} \subseteq \Psi^{\prime},\{A\} \cup \square \Psi_{2}^{\prime}$ is a finite subset of $\Psi$. It follows that $\Psi$ is not tab-consistent.

We now are ready to define the canonical interpretation of a formula set.

Definition 11 Let $\Psi$ be a finite set of closed formulas and let the initial set $(W, 0): S^{(W, 0)}$ be tab-consistent. The canonical interpretation of $S^{(W, 0)}$ is a tuple $M_{c}=\left(W, \vec{w}_{0},\left\{R_{1}, \ldots, R_{m}\right\}, R, U, I\right)$ fulfilling the following conditions:

1. $W$ is a set of $m$-tuples for labeled sets of closed formulas where 
(a) $\vec{w}_{0} \in W$ with $\vec{w}_{0}=\langle 1, \ldots, m\rangle$ for $(W, 0):\left(S^{(W, 0)}\right)^{*}$ such that $\left(S^{(W, 0)}\right)^{*}$ is a superset of $S^{(W, 0)}, \vec{w}_{0}$ is saturated (it exists by Lemma 4), and $\max \left(\vec{w}_{0}\right)=m$. Then, $\vec{w}_{0}$ is tab-consistent since $(W, 0): S^{(W, 0)}$ is tab-consistent,

(b) if $\vec{w} \in W$ with $\vec{w}=\left\langle w_{1}, \ldots, w_{m}\right\rangle$ for $(i, n): \Psi_{k}(n>0)$, then

i. for every $\diamond_{i} A$ in $\Psi_{k}\left(=\left\{\diamond_{i} A\right\} \cup \square_{i} \Psi \backslash \mathbf{\square} \Psi^{\prime} \cup \Psi^{\prime \prime}\right), \vec{w}^{\prime}=\left\langle w_{1}, \ldots, w_{i-1}\right.$, $\left.\max (\vec{w})+1, w_{i+1}, \ldots, w_{m}\right\rangle$ for $(i, n+1): \Psi_{k}^{\prime}$ such that $\Psi_{k}^{\prime}$ is a superset of $\{A \wedge \mathcal{E}(A)\} \cup\left(\Psi^{\prime} \vee \neg \mathcal{E}\left(\Psi^{\prime}\right)\right) \cup \square_{i} \Psi^{\prime} \cup \square \Psi^{\prime}$, $\vec{w}^{\prime}$ is saturated, $\max \left(\vec{w}^{\prime}\right)=\max (\vec{w})+1,(i, n+1):\{A \wedge \mathcal{E}(A)\} \cup\left(\Psi^{\prime} \vee\right.$ $\left.\neg \mathcal{E}\left(\Psi^{\prime}\right)\right) \cup \square_{i} \Psi^{\prime} \cup \boldsymbol{\square} \Psi^{\prime}$ is tab-consistent (by Lemma 5), $\vec{w}^{\prime}$ exists and is tab-consistent (by Lemma 4), and $\left\langle\vec{w}, \vec{w}^{\prime}\right\rangle \in \mathbf{R}_{i}$,

ii. for every $\diamond_{j} A(i \neq j)$ in $\Psi_{k}\left(=\left\{\diamond_{j} A\right\} \cup \square_{j} \Psi^{\prime} \cup \mathbf{\square} \Psi^{\prime} \cup \Psi^{\prime \prime}\right)$, $\vec{w}^{\prime}=\left\langle w_{1}, \ldots, w_{j-1}, \max (\vec{w})+1, w_{j+1}, \ldots, w_{m}\right\rangle$ for $(j, n+1): \Psi_{k}^{\prime}$ such that $\Psi_{k}^{\prime}$ is a superset of $\{A \wedge \mathcal{E}(A)\} \cup\left(\Psi^{\prime} \vee \neg \mathcal{E}\left(\Psi^{\prime}\right)\right) \cup \mathbf{\square} \Psi^{\prime}$, $\vec{w}^{\prime}$ is saturated, $\max \left(\vec{w}^{\prime}\right)=\max (\vec{w})+1,(j, n+1):\{A \wedge \mathcal{E}(A)\} \cup$ $\left(\Psi^{\prime} \vee \neg \mathcal{E}\left(\Psi^{\prime}\right)\right) \cup \Psi^{\prime}$ is tab-consistent (by Lemma 5), $\vec{w}^{\prime}$ exists and is tab-consistent (by Lemma 4), and $\left\langle\vec{w}, \vec{w}^{\prime}\right\rangle \in \mathbf{R}_{j}$,

iii. for every $\wedge$ in $\Psi_{k}\left(=\{\backslash A\} \cup \mathbf{\square} \Psi^{\prime} \cup \Psi^{\prime \prime}\right), \vec{w}^{\prime}=\langle\max (\vec{w})+$ $1, \ldots, \max (\vec{w})+m\rangle$ for $(W, n+1): \Psi_{k}^{\prime}$ such that $\Psi_{k}^{\prime}$ is a superset of $\{A \wedge \mathcal{E}(A)\} \cup\left(\Psi^{\prime} \vee \neg \mathcal{E}\left(\Psi^{\prime}\right)\right) \cup \boldsymbol{\nabla} \Psi^{\prime}, \vec{w}^{\prime}$ is saturated, $\max \left(\vec{w}^{\prime}\right)=$ $\max (\vec{w})+m,(W, n+1):\{A \wedge \mathcal{E}(A)\} \cup\left(\Psi^{\prime} \vee \neg \mathcal{E}\left(\Psi^{\prime}\right)\right) \cup \mathbf{\square} \Psi^{\prime}$ is tab-consistent (by Lemma 5), $\vec{w}^{\prime}$ exists and is tab-consistent (by Lemma 4), and $\left\langle\vec{w}, \vec{w}^{\prime}\right\rangle \in \mathbf{R}^{\prime}$;

2. $W_{i}=\left\{w_{i} \mid \vec{w}=\left\langle w_{1}, \ldots, w_{m}\right\rangle\right.$ for $\left.(i, n): \Psi_{k}\right\}$;

3. $R_{i}=\operatorname{cl}\left(\mathbf{R}_{i}\right)$ or $\mathrm{cl}^{+}\left(\mathbf{R}_{i}\right)^{2}$;

4. $R=\operatorname{cl}\left(R_{1} \cup \cdots \cup R_{m} \cup \mathbf{R}^{\prime}\right)$;

5. $U$ is a superset of $\bigcup_{\vec{w} \in W} U_{\vec{w}}$ where $U_{\vec{w}}=\left\{t \in \mathcal{T}_{0} \mid t^{(i, n)}\right.$ occurs in $\left.\Psi_{k}\right\}$ and $\vec{w}$ is for $(i, n): \Psi_{k}$;

6. I is the set of interpretation functions $I_{\vec{w}}$ for all worlds $\vec{w} \in W$ for $(i, n): \Psi_{k}$ such that

(a) $I_{\vec{w}}(s)=\mathcal{T}_{s, 0}\left((i, n): \Psi_{k}\right) \cup \mathcal{T}_{p_{s}, 0}\left((i, n): \Psi_{k}\right)$,

(b) $I_{\vec{w}}(c)=c_{\tau}$ where $c: \rightarrow \tau \in \Omega$,

(c) $I_{\vec{w}}(f)\left(t_{1}, \ldots, t_{l}\right)=f_{\tau^{*}, \tau}\left(t_{1}, \ldots, t_{l}\right)$ with $\tau^{*}=\tau_{1}, \ldots, \tau_{l}$ where $f: \tau_{1} \times$ $\cdots \times \tau_{l} \rightarrow \tau \in \Omega$,

\footnotetext{
${ }^{2}$ Let $r$ be an ordered set. $c l(r)$ (resp. $\left.c l^{+}(r)\right)$ denotes the reflexive and transitive closure of $r$ (resp. the reflexive, transitive, and symmetric closure of $r$ ). This is used to make an accessibility relation reflexive and transitive (or reflexive, transitive, and symmetric) over possible worlds.
} 
(d) $I_{\vec{w}}(p)=\left\{\left(t_{1}, \ldots, t_{l}\right) \mid p\left(t_{1}^{(i, n)}, \ldots, t_{l}^{(i, n)}\right) \in \Psi_{k}\right\}$.

Let $\Psi$ be a finite set of closed formulas such that the initial tableau $(W, 0): \Psi^{(W, 0)}$ is tab-consistent. The following lemma indicates model existence for $\Psi$ and the semantic condition of the existential rigidity holding.

Lemma 6 Let $(W, 0): \Psi^{(W, 0)}$ be tab-consistent and let $M_{c}=\left(W, \vec{w}_{0},\left\{R_{1}, \ldots, R_{m}\right\}\right.$, $R, U, I)$ be the canonical interpretation of $\Psi^{(W, 0)}$. (i) $M_{c}$ is a sorted $\Sigma$-structure with existential rigidity, and (ii) for all $F \in\left(S^{(W, 0)}\right)^{*}$ where $\vec{w}_{0}$ is for $(W, 0):\left(S^{(W, 0)}\right)^{*}$, $\vec{w}_{0} \models F^{\prime}$ where $F^{\prime}$ is the non-annotated formula of $F$.

Proof. Let $M_{c}=\left(W, \vec{w}_{0},\left\{R_{1}, \ldots, R_{m}\right\}, R, U, I\right)$ be the canonical interpretation of $\Psi^{(W, 0)}$.

(i) We show that $M_{c}$ satisfies Conditions (1) - (5) in the definition of sorted $\Sigma$-structures and Conditions (1) - (4) in the definition of existential rigidity (i.e., sorted $\Sigma$-structures with existential rigidity).

(sorted $\Sigma$-structure in Definition 6) The dummy constants make the domain of each world non-empty. So, by Definition 11(1) - (2), Conditions (1) and (2) are satisfied. By Definition 11 (3), Condition (3) is satisfied, and by Definition 11 (4), Condition (4) is satisfied. By Definition 11 (5), Condition (5) is satisfied.

Condition (6-a). Let $s \in T \cup S_{A}$. By Definition $11(5)$ and (6-a), $I_{\vec{w}}(s)=$ $\mathcal{T}_{s, 0}(\vec{w}) \cup \mathcal{T}_{p_{s}, 0}(\vec{w}) \subseteq U_{\vec{w}}$.

Condition (6-b). Let $t \in I_{\vec{w}}(s)$ with $s \leq s^{\prime}$. By Definition 11 (6-a), $t \in$ $\mathcal{T}_{s, 0}(\vec{w}) \cup \mathcal{T}_{p_{s}, 0}(\vec{w})\left(\subseteq U_{\vec{w}}\right)$. By Lemma 3 , if $t \in \mathcal{T}_{s, 0}(\vec{w})$ then $t \in \mathcal{T}_{s^{\prime}, 0}(\vec{w})$, and if $t \in \mathcal{T}_{p_{s}, 0}(\vec{w})$ then $t \in \mathcal{T}_{p_{s^{\prime}}, 0}(\vec{w})$. Thus, we have $t \in I_{\vec{w}}\left(s^{\prime}\right)$.

Condition (6-c). By Definition 11 (6-a) and (6-b), for every $c \in C$ with $c: \rightarrow \tau \in \Omega, I_{\vec{w}}(c)=c_{\tau} \in \mathcal{T}_{0} \cap \mathcal{T}_{\tau} \subseteq I_{\vec{w}}(\tau)$.

Condition (6-d). By Definition 11 (6-a) and (6-c), for every $f \in F_{n}$ with $f: \tau_{1} \times \cdots \times \tau_{l} \rightarrow \tau \in \Omega, I_{\vec{w}}(f)\left(t_{1}, \ldots, t_{l}\right)=f_{\tau^{*}, \tau}\left(t_{1}, \ldots, t_{l}\right) \in \mathcal{T}_{0} \cap \mathcal{T}_{\tau} \subseteq I_{\vec{w}}(\tau)$ where $\tau^{*}=\tau_{1}, \ldots, \tau_{n}$.

Condition (6-e). Let $\left(t_{1}, \ldots, t_{l}\right) \in I_{\vec{w}}(p)$ where $\vec{w}$ is for $(i, n): \Psi_{k}$ and $p: s_{1} \times$ ... $\times s_{l} \in \Omega$. By Definition $11(6-\mathrm{d}), p\left(t_{1}^{(i, n)}, \ldots, t_{l}^{(i, n)}\right) \in \Psi_{k}$ where $t_{u} \in \mathcal{T}_{0} \cap \mathcal{T}_{s_{u}}$ for all $u \in\{1, \ldots, l\}$. By Condition (6-a), $U_{\vec{w}} \cap I_{\vec{w}}(s) \subseteq I_{\vec{w}}(s)$ for every $s \in$ $T \cup S_{A}$. Hence, $I_{\vec{w}}(p) \subseteq I_{\vec{w}}\left(s_{1}\right) \times \cdots \times I_{\vec{w}}\left(s_{l}\right)$. Let $t \in I_{\vec{w}}\left(p_{s}\right)$ where $\vec{w}$ is for $(i, n): \Psi_{k}$ and $p_{s}: \tau \in \Omega$. By Definition $11(6-\mathrm{d}), p_{s}\left(t^{(i, n)}\right) \in \Psi_{k}$ where $t \in \mathcal{T}_{0} \cap \mathcal{T}_{\tau}$. So, by $(\mathrm{v}-1), t \in I_{\vec{w}}(\tau)$, and hence $I_{\vec{w}}\left(p_{s}\right) \subseteq I_{\vec{w}}(\tau)$ by Definition 11 (6-a).

Condition (6-f). Let $\vec{w} \in W$ for $(i, n): \Psi_{k}$ and let $t \in I_{\vec{w}}(s)$. Let $s=\tau$. If $t \in \mathcal{T}_{\tau, 0}(\vec{w})$, then by Definition $10(1), p_{\tau}\left(t^{(i, n)}\right) \in \Psi_{k}$. Let $s=\sigma$. Since there is no ground anti-rigid sorted term, $\mathcal{T}_{\sigma, 0}(\vec{w})=\emptyset$. On the other hand, if $t \in \mathcal{T}_{p_{s}, 0}(\vec{w})$, then by definition, $p_{s^{\prime}}\left(t^{(i, n)}\right) \in \Psi_{k}$ with $s^{\prime} \leq s$. When $s^{\prime} \neq s$, by Definition $10(2), p_{s}\left(t^{(i, n)}\right) \in \Psi_{k}$. Thus, $I_{\vec{w}}(s) \subseteq I_{\vec{w}}\left(p_{s}\right)$. If $\vec{w} \in W$ for 
$(i, n): \Psi_{k}$ and $t \in I_{\vec{w}}\left(p_{s}\right)$, then $p_{s}\left(t^{(i, n)}\right) \in \Psi_{k}$. By Definition 11 (6-a) and $(6-\mathrm{d}), t \in \mathcal{T}_{p_{s}, 0}(\vec{w})\left(\subseteq I_{\vec{w}}(s)\right)$. From this, $I_{\vec{w}}\left(p_{s}\right) \subseteq I_{\vec{w}}(s)$ follows.

(existential rigidity in Definition 7) Conditions (1) and (2). Let $\vec{w}_{i}, \vec{w}_{j} \in W$ and let $c_{\tau}$ or $f_{\tau^{*}, \tau}\left(t_{1}, \ldots, t_{l}\right)$ in $U_{\vec{w}_{i}} \cap U_{\vec{w}_{j}}$. Definition 11 (6-b) entails $I_{\vec{w}_{i}}(c)=$ $c_{\tau}=I_{\vec{w}_{j}}(c)$ and $I_{\vec{w}_{i}}(f)\left(t_{1}, \ldots, t_{l}\right)=f_{\tau^{*}, \tau}\left(t_{1}, \ldots, t_{l}\right)=I_{\vec{w}_{j}}(f)\left(t_{1}, \ldots, t_{l}\right)$ where $c: \rightarrow \tau \in \Omega$ and $f: \tau_{1} \times \cdots \times \tau_{l} \rightarrow \tau \in \Omega$.

Condition (3). Let $w \in W$ and $t \in U_{\vec{w}}$. Let $\tau$ be a type with $t \in I_{\vec{w}}(\tau)(=$ $\left.\mathcal{T}_{\tau, 0}\left((i, n): \Psi_{k}\right) \cup \mathcal{T}_{p_{\tau}, 0}\left((i, n): \Psi_{k}\right)\right)$. If $t \in \mathcal{T}_{\tau, 0}\left((i, n): \Psi_{k}\right)$, then by Definition 10 (1), $p_{\tau}\left(t^{(i, n)}\right) \in \Psi_{k}$. If $t \in \mathcal{T}_{p_{\tau}, 0}\left((i, n): \Psi_{k}\right)$, then $p_{s}\left(t^{(i, n)}\right) \in \Psi_{k}$ with $s \leq \tau$. So, by Definition $10(2), p_{\tau}\left(t^{(i, n)}\right) \in \Psi_{k}$. Therefore, by Definition 10 (3), we have - $p_{\tau}(t) \in \Psi_{k}$. Let $\left\langle\vec{w}, \vec{w}^{\prime}\right\rangle \in R$. Then, by Definition $11(1-\mathrm{b}), p_{\tau}(t) \vee \neg \mathcal{E}\left(p_{\tau}(t)\right) \in$ $\Psi_{k}^{\prime}$ where $\vec{w}^{\prime}$ is for $\left(i^{\prime}, n+m\right): \Psi_{k}^{\prime}$. If $t \in U_{\vec{w}^{\prime}}$ (iff $\left.\neg E(t) \notin \Psi_{k}^{\prime}\right)$, then $p_{\tau}\left(t^{n+m}\right)$ must belong to $\Psi_{k}^{\prime}$. So, $t \in \mathcal{T}_{p_{\tau}, 0}\left(\left(i^{\prime}, n+m\right): \Psi_{k}^{\prime}\right)$. Therefore, by Definition 11 (6-a), $t \in I_{\vec{w}^{\prime}}(\tau)\left(=\mathcal{T}_{\tau, 0}\left(\left(i^{\prime}, n+m\right): \Psi_{k}^{\prime}\right) \cup \mathcal{T}_{p_{\tau}, 0}\left(\left(i^{\prime}, n+m\right): \Psi_{k}^{\prime}\right)\right)$.

Condition (4). Let $\vec{w} \in W$ and $t \in U_{\vec{w}}$. Let $\sigma$ be an anti-rigid sort with $t \in$ $I_{\vec{w}}(\sigma)\left(=\mathcal{T}_{\sigma, 0}\left((i, n): \Psi_{k}\right) \cup \mathcal{T}_{p_{\sigma}, 0}\left((i, n): \Psi_{k}\right)\right)$ where $\vec{w}$ is for $(i, n): \Psi_{k}$. Since there is no ground anti-rigid sorted term, $\mathcal{T}_{\sigma, 0}\left((i, n): \Psi_{k}\right)=\emptyset$. If $t \in \mathcal{T}_{p_{\sigma}, 0}\left((i, n): \Psi_{k}\right)$, then by definition, $p_{\sigma^{\prime}}\left(t^{(i, n)}\right) \in \Psi_{k}$ with $\sigma^{\prime} \leq \sigma$. When $\sigma^{\prime} \neq \sigma$, by Definition 10 (2), $p_{\sigma}\left(t^{(i, n)}\right) \in \Psi_{k}$. Then, by Definition $10(4), \neg p_{\sigma}\left(t^{\prime}\right) \in \Psi_{k}$. By Definition 11 (1-b), there exists $\vec{w}^{\prime}$ for $(W, n+1): \Psi_{k}^{\prime}$ such that $\neg p_{\sigma}(t), E\left(t^{(W, n+1)}\right) \in \Psi_{k}^{\prime}$. So, $p_{\sigma}\left(t^{(W, n+1)}\right) \notin \Psi_{k}^{\prime}$. Hence, $t \notin \mathcal{T}_{p_{\sigma}, 0}\left((W, n+1): \Psi_{k}^{\prime}\right)$. Since $\mathcal{T}_{\sigma, 0}((W, n+$ $\left.1): \Psi_{k}^{\prime}\right)=\left\{a_{(W, n+1)}\right\}$ and $t \neq a_{(W, n+1)}$, we have $t \notin \mathcal{T}_{\sigma, 0}\left(\vec{w}^{\prime}\right)$. Therefore, by Definition 11 (6-a), $t \notin I_{\vec{w}^{\prime}}(\sigma)$ with $t \in U_{\vec{w}^{\prime}}$.

(ii) We next verify that for every $\vec{w} \in W$ for $(i, n): \Psi_{k}$ and for every $F \in \Psi_{k}$, $\vec{w}=F^{\prime}$ where $F^{\prime}$ is the non-annotated formula of $F$.

Let $F=p\left(t_{1}^{(i, n)}, \ldots, t_{l}^{(i, n)}\right)$. Due to $p\left(t_{1}^{(i, n)}, \ldots, t_{l}^{(i, n)}\right) \in \Psi_{k}$, it is clear that $\vec{w}=p\left(t_{1}, \ldots, t_{l}\right)$ (by Definition $11(6-\mathrm{d})$ ).

Let $F=\neg p\left(t_{1}, \ldots, t_{l}\right)$. Since $(i, n): \Psi_{k}$ is tab-consistent, $p\left(t_{1}^{(i, n)}, \ldots, t_{l}^{(i, n)}\right) \notin$ $\Psi_{k}$. Thus, $\vec{w} \mid \neq p\left(t_{1}, \ldots, t_{l}\right)$.

Let $F=F_{1} \wedge F_{2}$. Then, $F_{1}^{(i, n)}, F_{2}^{(i, n)} \in \Psi_{k}$ because $\vec{w}$ is saturated. By the induction hypothesis, $\vec{w}=F_{1}$ and $\vec{w} \models F_{2}$. So, $\vec{w}=F_{1} \wedge F_{2}$.

Let $F=F_{1} \vee F_{2}$. Then, $F_{1}^{(i, n)} \in \Psi_{k}$ or $F_{2}^{(i, n)} \in \Psi_{k}$ since $\vec{w}$ is saturated. By the induction hypothesis, $\vec{w} \models F_{1}$ or $\vec{w} \models F_{2}$. Hence, $\vec{w}=F_{1} \vee F_{2}$.

Let $F=\left(\forall x_{s}\right) F_{1}$. By Definition $10(5), F_{1}\left[x_{s} / t\right]^{(i, n)} \in \Psi_{k}$ for every ground term $t \in \mathcal{T}_{s, 0}(\vec{w}) \cup \mathcal{T}_{p_{s}, 0}(\vec{w})\left(=I_{\vec{w}}(s)\right)$. By the induction hypothesis, $\vec{w}=F_{1}\left[x_{s} / t\right]$. This yields $\vec{w}=\left(\forall x_{s}\right) F_{1}$.

Let $F=\left(\exists x_{s}\right) F_{1}$. By Definition $10(6), p_{s}\left(c_{\tau}^{(i, n)}\right) \in \Psi_{k}$ and $F_{1}\left[x_{s} / c_{\tau}\right]^{(i, n)} \in$ $\Psi_{k}$ for some constant $c_{\tau}$. If $s \in T$, then $s=\tau$. Thus, $c_{\tau} \in \mathcal{T}_{0} \cap \mathcal{T}_{\tau}$ and it occurs in $\Psi_{k}$. This implies $c_{\tau} \in \mathcal{T}_{s, 0}(\vec{w})\left(\subseteq I_{\vec{w}}(s)\right)$. If $s \notin T$, then $p_{s}$ must be inextensible (since $s$ is an anti-rigid sort). Then, $c_{\tau} \in \mathcal{T}_{p_{s}, 0}(\vec{w})\left(\subseteq I_{\vec{w}}(s)\right)$. By the induction hypothesis, $\vec{w} \models F_{1}\left[x_{s} / c_{\tau}\right]$. Hence, $\vec{w}=\left(\exists x_{s}\right) F_{1}$. 
Let $F=\square_{j} F_{1}$. Let $\vec{w}^{\prime} \in W$ such that $\left\langle\vec{w}, \vec{w}^{\prime}\right\rangle \in R_{j}$ and $\vec{w}^{\prime}$ is for $(j, n+$ $m): \Psi_{k}^{\prime}$. By Definition 11 (1-b)-i and ii, $F_{1} \vee \neg \mathcal{E}\left(F_{1}\right) \in \Psi_{k}^{\prime}$. By Definition 10 (4), $F_{1}^{n+m} \in \Psi_{k}^{\prime}$ or $\neg \mathcal{E}\left(F_{1}\right) \in \Psi_{k}^{\prime}$ (if $\mathcal{E}\left(F_{1}\right) \neq \emptyset$ ). By the induction hypothesis, if $F \notin N e x_{\vec{w}^{\prime}}$, then $\vec{w}^{\prime} \models F_{1}$. Therefore, $\vec{w} \models \square_{j} F_{1}$.

Let $F=\diamond_{j} F_{1}$. By Definition 11 (1-b)-i and ii, there exists $\vec{w}^{\prime}$ for $(j, n+$ 1): $\Psi_{k}^{\prime}$ such that $\left\langle\vec{w}, \vec{w}^{\prime}\right\rangle \in R_{j}$ and $F_{1} \wedge \mathcal{E}\left(F_{1}\right) \in \Psi_{k}^{\prime}$. Since $\Psi_{k}^{\prime}$ is saturated, $F_{1} \in \Psi_{k}^{\prime}$ and $\mathcal{E}\left(F_{1}\right) \in \Psi_{k}^{\prime}$ (if $\mathcal{E}\left(F_{1}\right) \neq \emptyset$ ). By the induction hypothesis, $\vec{w}^{\prime}=F_{1}$ and by Lemma 1 (1), $F_{1} \notin N e x_{\vec{w}^{\prime}}$. By definition, $\vec{w} \models \diamond_{j} F_{1}$.

Let $F=\boldsymbol{\square} F_{1}$. Let $\vec{w}^{\prime} \in W$ such that $\left\langle\vec{w}, \vec{w}^{\prime}\right\rangle \in R$ and $\vec{w}^{\prime}$ is for $\left(i^{\prime}, n+m\right): \Psi_{k}^{\prime}$. By Definition 11 (1-b)-iii, $F_{1} \vee \neg \mathcal{E}\left(F_{1}\right) \in \Psi_{k}^{\prime}$. By Definition $10(4), F_{1}^{n+m} \in \Psi_{k}^{\prime}$ or $\neg \mathcal{E}\left(F_{1}\right) \in \Psi_{k}^{\prime}$ (if $\mathcal{E}\left(F_{1}\right) \neq \emptyset$ ). By the induction hypothesis, if $F \notin N e x_{\vec{w}^{\prime}}$, then $\vec{w}^{\prime} \models F_{1}$. Hence, $\vec{w}=\mathbf{\square} F_{1}$.

Let $F=F_{1}$. By Definition $11(1-\mathrm{b})$-iii, there exists $\vec{w}^{\prime}$ for $(W, n+1): \Psi_{k}^{\prime}$ such that $\left\langle\vec{w}, \vec{w}^{\prime}\right\rangle \in R$ and $F_{1} \wedge \mathcal{E}\left(F_{1}\right) \in \Psi_{k}^{\prime}$. Since $\Psi_{k}^{\prime}$ is saturated, $F_{1} \in \Psi_{k}^{\prime}$ and $\mathcal{E}\left(F_{1}\right) \in \Psi_{k}^{\prime}\left(\right.$ if $\left.\mathcal{E}\left(F_{1}\right) \neq \emptyset\right)$. By the induction hypothesis, $\vec{w}^{\prime} \models F_{1}$ and by Lemma 1 (1), $F_{1} \notin N e x_{\vec{w}^{\prime}}$. Therefore, $\vec{w}=F_{1}$.

Theorem 2 If $\Psi$ is $\Sigma$-unsatisfiable, then there exists a closed tableau for $\Psi$.

Proof. We show that if there exists no closed tableau for $\Psi$, then $\Psi$ is $\Sigma$-satisfiable. By the assumption, there is no subset $\Psi^{\prime}$ of $S^{(W, 0)}$ such that $(W, 0): \Psi^{\prime}$ has a closed tableau, i.e., $(W, 0): S^{(W, 0)}$ is tab-consistent. So, by Definition $11, \Psi^{(W, 0)} \subseteq S^{(W, 0)} \subseteq\left(S^{(W, 0)}\right)^{*}$ where $\vec{w}_{0}$ is for $(W, 0):\left(S^{(W, 0)}\right)^{*}$, and by Lemma $6, \vec{w}_{0}=\Psi$. Thus, it is $\Sigma$-satisfiable.

This theorem leads to the completeness of the tableau calculus as follows:

Theorem 3 (Completeness) There exists a closed tableau for $\Psi$ if and only if $\Psi$ is $\Sigma$-unsatisfiable.

Proof. By Theorem 1 and Theorem 2, it can be proved.

\subsection{Examples}

We consider three examples for testing the consequence and validity of sorted modal formulas using the proposed tableau calculus.

Example 1 Let $\Sigma_{1}=\left(T, S_{A}, \leq, \Omega\right)$ be a sorted signature such that

1. $T=\{$ man, person, $\top\}$,

2. $S_{A}=\emptyset$, 
3. $\leq$ is the transitive and reflexive closure of $\{($ man, person $),($ person, $\top)\}$, $=\left\{p_{\text {man }}, p_{\text {person }}, p_{\top}\right\}$,

4. $\Omega=\left\{\right.$ bob: $\rightarrow$ person, $\left.p_{\text {man }}: \top, p_{\text {person }}: \top, p_{\top}: \top\right\}$.

We prove that the following sorted modal formula $F_{1}$ is a consequence of $\Psi_{1}$ by using the calculus.

$$
\begin{aligned}
& F_{1}=p_{\text {man }}\left(\text { bob }_{\text {person }}\right) \rightarrow \boldsymbol{\square}_{\text {man }}\left(\text { bob }_{\text {person }}\right) \\
& \Psi_{1}=\left\{\boldsymbol{\square} \forall x_{\text {person }}\left(p_{\text {man }}\left(x_{\text {person }}\right) \rightarrow p_{\text {man }}\left(x_{\text {person }}\right)\right)\right\}
\end{aligned}
$$

The formula $F_{1}$ indicates that if $b o b_{\text {person }}$ is a man, then he is a man in any world as long as it exists. The formula set $\Psi_{1}$ states that every man is always a man in any possible world.

In order to test the consequence $\Psi_{1} \models F_{1}$, it is sufficient to check the unsatisfiability of $\left\{\neg F_{1}\right\} \cup \Psi_{1}$ since $\Psi_{1} \models F_{1}$ holds if and only if $\left\{\neg F_{1}\right\} \cup \Psi_{1}$ is $\Sigma$-unsatisfiable. According to Proposition 1, the formulas in $\left\{\neg F_{1}\right\} \cup \Psi_{1}$ are transformed into equivalent ones in negation normal form as follows:

$$
\begin{aligned}
& \neg F_{1}=\neg\left(p_{\text {man }}\left(b_{o b} b_{\text {person }}\right) \rightarrow p_{\text {man }}\left(b_{o b} b_{\text {person }}\right)\right) \\
& \simeq p_{\text {man }}\left(\text { bob }_{\text {person }}\right) \wedge \neg p_{\text {man }}\left(\text { bob }_{\text {person }}\right) \\
& \simeq p_{\text {man }}\left(\text { bob }_{\text {person }}\right) \wedge \wedge\left(\neg p_{\text {man }}\left(\text { bob }_{\text {person }}\right)\right) \\
& \Psi_{1}=\left\{\boldsymbol{\square} \forall x_{\text {person }}\left(p_{\text {man }}\left(x_{\text {person }}\right) \rightarrow \boldsymbol{\square}_{\text {man }}\left(x_{\text {person }}\right)\right)\right\} \\
& \simeq\left\{\boldsymbol{\square} \forall x_{\text {person }}\left(\neg p_{\text {man }}\left(x_{\text {person }}\right) \vee \boldsymbol{D}_{\text {man }}\left(x_{\text {person }}\right)\right)\right\}
\end{aligned}
$$

Figure 2 illustrates a proof of testing the unsatisfiability of $\left\{\neg F_{1}\right\} \cup \Psi_{1}$ where every tableau for $\left\{\neg F_{1}\right\} \cup \Psi_{1}$ is closed. We abbreviate $p_{\text {man }}$ and bob person as $p_{m}$ and $b o b_{p}$, respectively. In Figure 2, the initial tableau of the normal negation form of $\left\{\neg F_{1}\right\} \cup \Psi_{1}$ :

$$
(W, 0): p_{m}\left(b_{p}\right) \wedge \triangleleft\left(\neg p_{m}\left(b_{p}\right)\right), \boldsymbol{\square} \forall x_{p}\left(\neg p_{m}\left(x_{p}\right) \vee \mathbf{\square}_{m}\left(x_{p}\right)\right)
$$

is decomposed to the following node by applying $\square$-rule, $\alpha$-rule, and $\gamma_{\tau}$-rule.

$$
(W, 0): p_{m}\left(b_{p}^{(W, 0)}\right), \diamond\left(\neg p_{m}\left(b_{p}\right)\right), \neg p_{m}\left(b_{p}\right) \vee \mathbf{\square}_{m}\left(b_{p}\right)
$$

This splits into the following two branch nodes:

$$
(W, 0): p_{m}\left(b_{p}^{(W, 0)}\right), \neg p_{m}\left(b_{p}\right), \triangleleft\left(\neg p_{m}\left(b_{p}\right)\right)
$$

and

$$
(W, 0): p_{m}\left(b_{p}^{(W, 0)}\right), \diamond\left(\neg p_{m}\left(b_{p}\right)\right), \mathbf{\square}_{m}\left(b_{p}\right)
$$

by applying $\beta$-rule to the disjunctive formula $\neg p_{m}\left(b_{p}\right) \vee q_{m}\left(b_{p}\right)$. The first branch is closed because it contains $p_{m}\left(b_{p}^{(W, 0)}\right)$ and $\neg p_{m}\left(b_{p}\right)$. The second branch is decomposed to the following node by applying $\pi_{W}$-rule and $\alpha$-rule.

$$
(W, 1): \neg p_{m}\left(b_{p}\right), E\left(b_{p}^{(W, 1)}\right), p_{m}\left(b_{p}\right) \vee \neg E\left(b_{p}\right)
$$




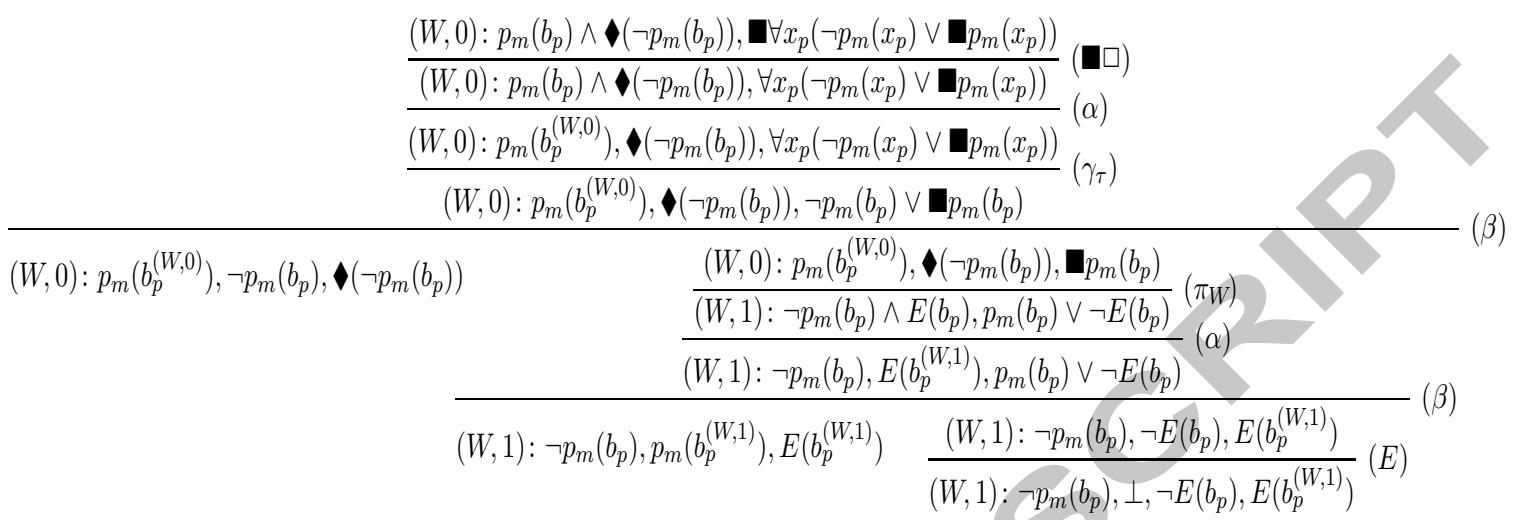

Figure 2: A proof for unsatisfiability of $\left\{\neg F_{1}\right\} \cup \Psi_{1}$

Furthermore, this splits into the following two branch nodes:

$$
(W, 1): \neg p_{m}\left(b_{p}\right), p_{m}\left(b_{p}^{(W, 1)}\right), E\left(b_{p}^{(W, 1)}\right)
$$

and

$$
(W, 1): \neg p_{m}\left(b_{p}\right), \neg E\left(b_{p}\right), E\left(b_{p}^{(W, 1)}\right)
$$

by applying $\beta$-rule to the disjunctive formula $p_{m}\left(b_{p}\right) \vee \neg E\left(b_{p}\right)$. The first branch is closed and the second branch derives the following closed node:

$$
(W, 1): \neg p_{m}\left(b_{p}\right), \perp, \neg E\left(b_{p}\right), E\left(b_{p}^{(W, 1)}\right)
$$

by applying $E$-rule. This concludes that $\left\{\neg F_{1}\right\} \cup \Psi_{1}$ is $\Sigma$-unsatisfiable because every leaf node in the tableau is closed, and hence $\Psi_{1} \models F_{1}$ holds.

Example 2 Let $\Sigma_{2}=\left(T, S_{A}, \leq, \Omega\right)$ be a sorted signature such that

1. $T=\{$ person, male, female, animal, $\top\}$,

2. $S_{A}=\{$ hospitalPatient, patient, doctor, nurse $\}$,

$3 . \leq$ is the transitive and reflexive closure of $\{$ (hospitalPatient,patient), (patient, person), (doctor, person), (nourse, person), (person, animal), (female, animal), (male, animal), (animal, $\top)\}$,

4. $\Omega=\left\{\right.$ bob: $\rightarrow$ person, $p_{\text {person }}: \top, p_{\text {hospitalPatient }}: \top, p_{\text {patient }}: \top, p_{\text {doctor }}:$ $\left.\top, p_{\text {nurse }}: \top, p_{\text {male }}: \top, p_{\text {female }}: \top, p_{\text {animal }}: \top, p_{\top}: \top\right\}$.

In the sorted signature, person, male, female, animal, and $\top$ are rigid sorts and hospitalPatient, patient, doctor, and nurse are anti-rigid sorts. 
We consider testing whether the following sorted modal formula $F_{2}$ is a consequence of $\Psi_{2}$ :

$$
\begin{aligned}
F_{2} & =\diamond_{\operatorname{Tim}} p_{\text {hospitalPatient }}\left(\text { bob }_{\text {person }}\right) \rightarrow \diamond_{\operatorname{Tim}}\left(\exists x_{\text {person }}\right)\left(\square_{\text {Sit }} p_{\text {patient }}\left(x_{\text {person }}\right)\right) \\
\Psi_{2} & =\left\{\square_{\operatorname{Tim}}\left(\forall y_{\text {person }}\right)\left(p_{\text {hospitalPatient }}\left(y_{\text {person }}\right) \rightarrow \square_{\text {Sit }} p_{\text {hospitalPatient }}\left(y_{\text {person }}\right)\right)\right\}
\end{aligned}
$$

The formula $F_{2}$ means that if Bob is a hospital patient at a time, a person exists at a time who is a patient in any situation within the time. The formula set $\Psi_{2}$ states that for every time of any possible world, every hospital patient is a hospital patient in any situation. The formulas in $\left\{\neg F_{2}\right\} \cup \Psi_{2}$ are transformed into equivalent ones in negation normal form as follows:

$$
\begin{aligned}
\neg F_{2} & =\neg\left(\diamond_{\operatorname{Tim}} p_{\text {hospitalPatient }}\left(\text { bob }_{\text {person }}\right) \rightarrow \diamond_{\operatorname{Tim}}\left(\exists x_{\text {person }}\right)\left(\square_{\text {Sit }} p_{\text {patient }}\left(x_{\text {person }}\right)\right)\right) \\
& \simeq \diamond_{\operatorname{Tim}} p_{\text {hospitalPatient }}\left(\text { bob }_{\text {person }}\right) \wedge \neg \diamond_{\operatorname{Tim}}\left(\exists x_{\text {person }}\right)\left(\square_{\text {Sit }} p_{\text {patient }}\left(x_{\text {person }}\right)\right) \\
& \simeq \diamond_{\operatorname{Tim}} p_{\text {hospitalPatient }}\left(\text { bob } b_{\text {person }}\right) \wedge \square_{\operatorname{Tim}} \neg\left(\exists x_{\text {person }}\right)\left(\square_{\operatorname{Sit}} p_{\text {patient }}\left(x_{\text {person }}\right)\right) \\
& \simeq \diamond_{\operatorname{Tim}} p_{\text {hospitalPatient }}\left(\text { bob } b_{\text {person }}\right) \wedge \square_{\operatorname{Tim}}\left(\forall x_{\text {person }}\right)\left(\neg \square \operatorname{Sit} p_{\text {patient }}\left(x_{\text {person }}\right)\right) \\
& \simeq \diamond_{\operatorname{Tim}} p_{\text {hospitalPatient }}(\text { bob person }) \wedge \square_{\operatorname{Tim}}\left(\forall x_{\text {person }}\right) \diamond_{\operatorname{Sit}}\left(\neg p_{\text {patient }}\left(x_{\text {person }}\right)\right) \\
\Psi_{2} & =\left\{\square_{\operatorname{Tim}}\left(\forall y_{\text {person }}\right)\left(p_{\text {hospitalPatient }}\left(y_{\text {person }}\right) \rightarrow \square_{\operatorname{Sit}} p_{\text {hospitalPatient }}\left(y_{\text {person }}\right)\right)\right\} \\
& \simeq\left\{\square_{\operatorname{Tim}}\left(\forall y_{\text {person }}\right)\left(\neg p_{\text {hospitalPatient }}\left(y_{\text {person }}\right) \vee \square_{\text {Sit }} p_{\text {hospitalPatient }}\left(y_{\text {person }}\right)\right)\right\}
\end{aligned}
$$

In Figure 3, we show a proof of testing the unsatisfiability of $\left\{\neg F_{2}\right\} \cup \Psi_{2}$. Since every tableau for $\left\{\neg F_{2}\right\} \cup \Psi_{2}$ is closed, $\Psi_{2} \models F_{2}$ holds. We abbreviate $p_{\text {hospitalPatient }}, p_{\text {patient }}, x_{\text {person }}, y_{\text {person }}$, and $b o b_{\text {person }}$ as $p_{h}, p_{a}, x_{p}, y_{p}$, and $b_{p}$, respectively. In Figure 2, the initial tableau of the normal negation form of $\left\{\neg F_{2}\right\} \cup \Psi_{2}$ :

$$
(W, 0): \diamond_{\operatorname{Tim}} p_{h}\left(b_{p}\right) \wedge \square_{\operatorname{Tim}}\left(\forall x_{p}\right) \diamond_{\mathbf{S i t}}\left(\neg p_{a}\left(x_{p}\right)\right), \square_{\mathbf{T i m}}\left(\forall y_{p}\right)\left(\neg p_{h}\left(y_{p}\right) \vee \square_{\mathbf{S i t}} p_{h}\left(y_{p}\right)\right)
$$

is decomposed to the following three branch nodes by applying some rules.

$$
\begin{gathered}
(\operatorname{Tim}, 1): p_{h}\left(b_{p}^{(\text {Tim }, 1)}\right), \neg p_{h}\left(b_{p}\right), E\left(b_{p}^{(\text {Tim }, 1)}\right), \diamond_{\text {Sit }}\left(\neg p_{a}\left(b_{p}\right)\right) \\
(\text { Sit, } 2): \neg p_{a}\left(b_{p}\right), p_{a}\left(b_{p}^{(\text {Sit }, 2)}\right), E\left(b_{p}^{(\text {Sit }, 2)}\right) \\
(\text { Sit }, 2): \neg p_{a}\left(b_{p}\right), \perp, \neg E\left(b_{p}\right), E\left(b_{p}^{(\text {Sit }, 2)}\right)
\end{gathered}
$$

This concludes that $\left\{\neg F_{2}\right\} \cup \Psi_{2}$ is $\Sigma$-unsatisfiable because these three nodes in the tableau are closed, and hence $\Psi_{2}=F_{2}$ holds.

Example 3 Let $\Sigma_{3}=\left(T, S_{A}, \leq, \Omega\right)$ be a sorted signature such that

1. $T=\{$ person, male, female, animal, $\top\}$,

2. $S_{A}=\{$ hospitalPatient, patient, doctor, nurse $\}$, 


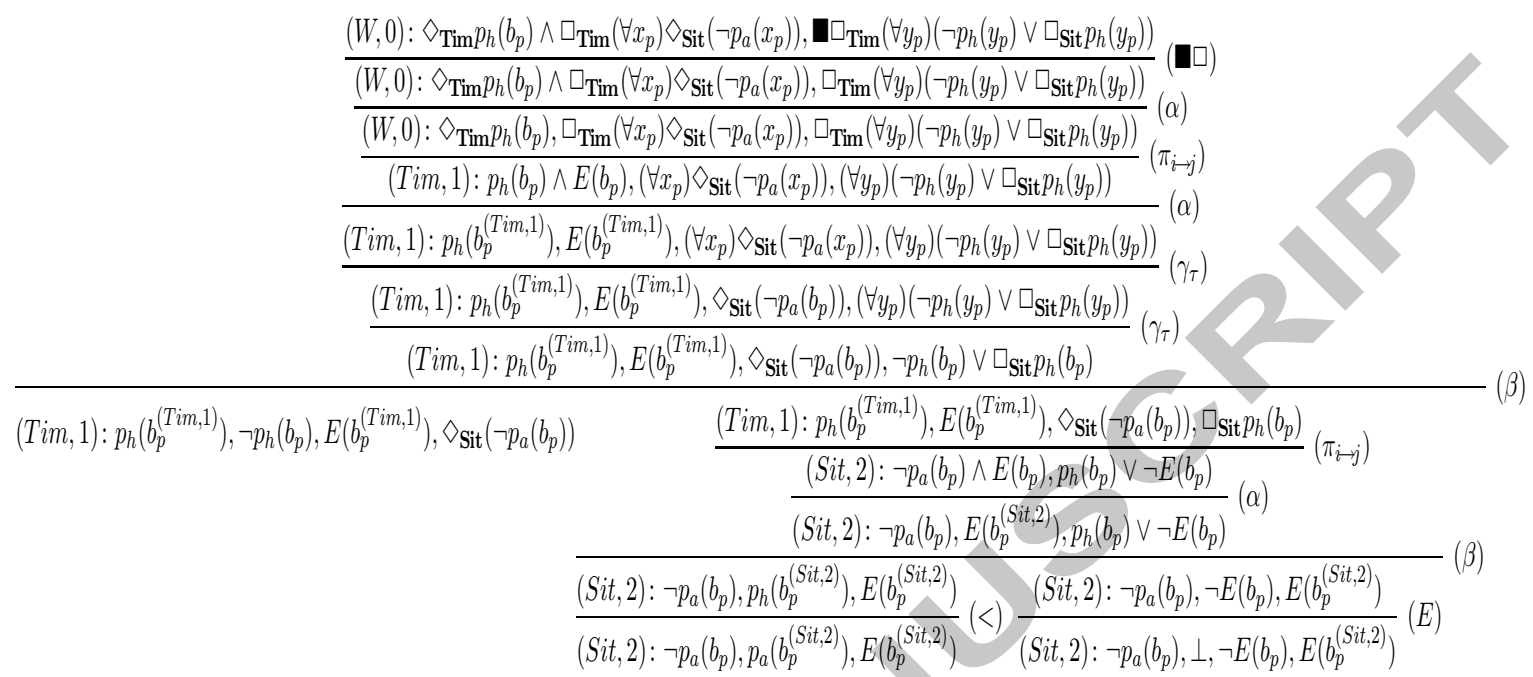

Figure 3: A proof for unsatisfiability of $\left\{\neg F_{2}\right\} \cup \Psi_{2}$

$3 . \leq$ is the transitive and reflexive closure of $\{$ (hospitalPatient,patient), (patient, person), (doctor, person), (nourse, person), (person, animal), (female, animal), (male, animal), (animal, $\top)\}$,

4. $\Omega=\left\{\right.$ bob: $\rightarrow$ person, $p_{\text {person }}: \top, p_{\text {hospitalPatient }}: \top, p_{\text {patient }}: \top, p_{\text {doctor }}$ : $\top, p_{\text {nurse }}: \top, p_{\text {male }}: \top, p_{\text {female }}: \top, p_{\text {animal }}: \top$, hypertension: person, maximumBloodPresure $120 \mathrm{mmHg}$ : T, minimumBloodPresure $80 \mathrm{~mm}$ $\left.H g: \top, p_{\top}: \top\right\}$.

In the sorted signature, we can represent the following formula:

$\left(\forall x_{\text {person }}\right)(($ maximumBloodPresure $120 \mathrm{mmHg}(x:$ person $) \vee$ minimumBloodPresure $80 \mathrm{mmHg}(x:$ person $)) \rightarrow$ hypertension $(x:$ person $))$

We consider testing the validity of the following sorted modal formula (the negative introspection axiom in epistemic logic):

$$
\begin{aligned}
& \left(\neg \square_{\mathbf{A g t 1}} \diamond_{\mathbf{T i m}} \text { hypertension }(\text { bob }: \text { person })\right) \rightarrow \\
& \quad\left(\square_{\mathbf{A g t 1} \mathbf{1}} \neg \square_{\mathbf{A g t 1}} \diamond_{\mathbf{T i m}} \text { hypertension }(\text { bob: person })\right)
\end{aligned}
$$

This states that an agent knows the fact "Bob has a hypertension at a time point" which s/he does not know. The formula $\neg F_{3}$ is transformed into an 


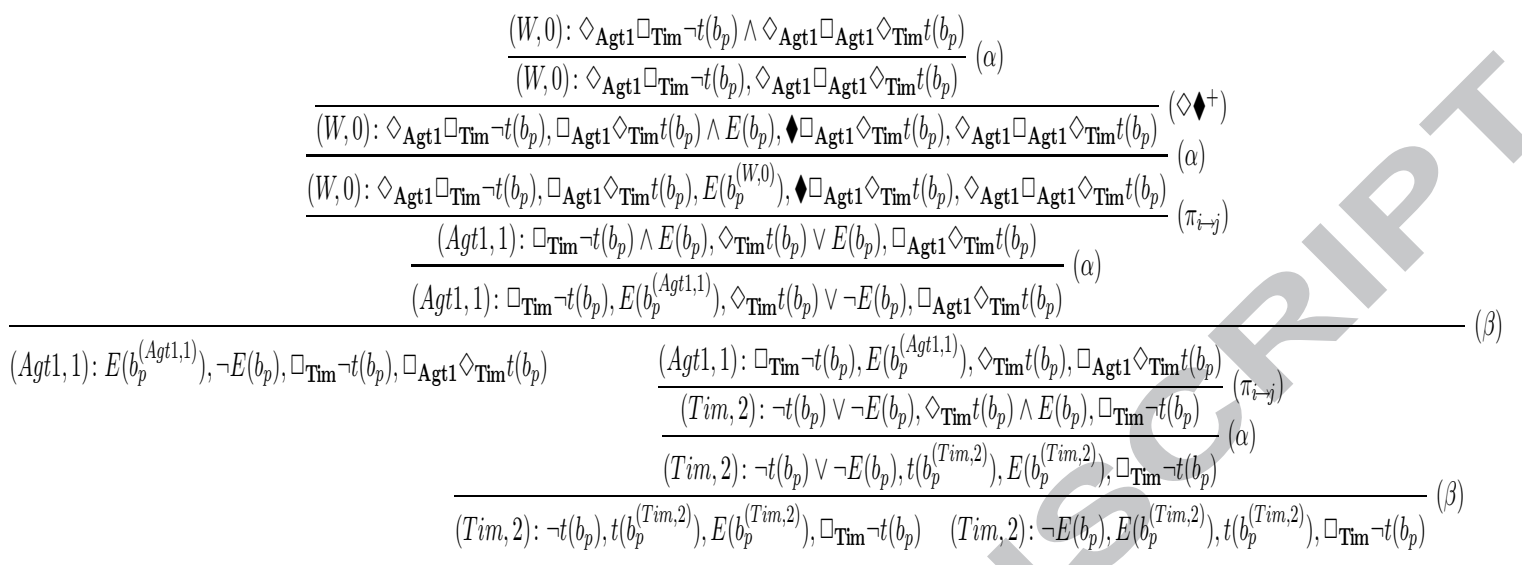

Figure 4: A proof for unsatisfiability of $\neg F_{3}$

equivalent one in negation normal form as follows:

$$
\begin{aligned}
& \neg\left(\neg \square_{\mathbf{A g t 1}} \diamond_{\text {Tim }} \text { hypertension }\left(b_{p}\right) \rightarrow \square_{\mathbf{A g t} 1} \neg \square_{\mathbf{A g t 1}} \diamond_{\text {Tim }} \text { hypertension }\left(b_{p}\right)\right) \\
& \simeq \neg \square_{\mathbf{A g t} \mathbf{1}} \diamond_{\mathbf{T i m}} \text { hypertension }\left(b_{p}\right) \wedge \neg \square \square_{\mathbf{A g t} \mathbf{1}} \neg \square_{\mathbf{A g t} \mathbf{1}} \diamond_{\mathbf{T i m}} \text { hypertension }\left(b_{p}\right) \\
& \simeq \diamond_{\mathbf{A g t 1}} \neg \diamond_{\mathbf{T i m}} \text { hypertension }\left(b_{p}\right) \wedge \neg \square \text { Agt1 } \neg \square_{\mathbf{A g t 1}} \diamond_{\mathbf{T i m}} \text { hypertension }\left(b_{p}\right) \\
& \simeq \diamond_{\mathbf{A g t 1} 1} \square_{\mathbf{T i m}} \neg \text { hypertension }\left(b_{p}\right) \wedge \neg \square_{\mathbf{A g t 1} 1} \neg \square_{\mathbf{A g t} \mathbf{1}} \diamond_{\mathbf{T i m}} \text { hypertension }\left(b_{p}\right) \\
& \simeq \diamond_{\mathbf{A g t 1} 1} \square_{\text {Tim }} \neg \text { hypertension }\left(b_{p}\right) \wedge \diamond_{\mathbf{A g t 1} 1} \neg \neg \square_{\mathbf{A g t 1}} \diamond_{\mathbf{T i m}} \text { hypertension }\left(b_{p}\right) \\
& \simeq \diamond_{\mathbf{A g t 1} 1} \square_{\mathbf{T i m}} \neg \text { hypertension }\left(b_{p}\right) \wedge \diamond_{\mathbf{A g t 1} 1} \square_{\mathbf{A g t} 1} \diamond_{\mathbf{T i m}} \text { hypertension }\left(b_{p}\right)
\end{aligned}
$$

In Figure 4, we show a proof of testing the unsatisfiability of the formula $\neg F_{3}$. Since every tableau for $\left\{\neg F_{3}\right\}$ is closed, $F_{3}$ is $\Sigma$-valid. We abbreviate hypertension and bob person as $t$ and $b_{p}$, respectively.

\section{Conclusion}

We have presented an extension of order-sorted logic where sorted modal formulas consist of rigid/anti-rigid sorted terms and many modal operators. The multi-modalities of sorted modal formulas are interpreted over the Cartesian product of sets of possible worlds. For the extended logic, we have developed a labeled tableau calculus that can test the satisfiability and validity of sorted modal formulas. In a previous study, Cialdea-Mayer and Cerrito's prefixed tableau calculus employed formulas labeled with a natural number $n$. In contrast, our tableau calculus is designed to handle many modalities together with the existential predicate and rigid/anti-rigid sorted terms. In particular, sorted modal formulas are labeled by the pair $(i, n)$ of the type of worlds $i$ and a natural number $n$ in the reasoning process using the proposed calculus. 
The proposed tableau calculus is useful for us in implementing a reasoning engine in various knowledge-based systems. In fact, many knowledge-based systems have to handle both dynamic knowledge in the real world and conceptual knowledge (e.g., ontology) in a specific domain because they may access and extract knowledge from various agents and networks. Our extension of ordersorted logic supports the rigidity and anti-rigidity of conceptual knowledge using many-dimensional modalities. The extended order-sorted logic provides a sound reasoning mechanism that can test the validity and logical consequence for the complex knowledge obtained by combining the dynamic knowledge and the conceptual ontology.

\section{References}

[1] C. Beierle, U. Hedtsück, U. Pletat, P.H. Schmitt, and J. Siekmann. An order-sorted logic for knowledge representation systems. Artificial Intelligence, 55:149-191, 1992.

[2] M. Cialdea-Mayer and S. Cerrito. Ground and free-variable tableaux for variants of quantified modal logics. Studia Logica, 69(1):97-131, 2001.

[3] A. G. Cohn. Taxonomic reasoning with many sorted logics. Artificial Intelligence Review, 3:89-128, 1989.

[4] R. P. Cruz and J. N. Crossley. Tiered logic for agents. In Proceedings of the International Conference on Agents and Artificial Intelligence (ICAART 2009), pages 369-376, 2009.

[5] M. Fitting and R. L. Mendelsohn. First-Order Modal Logic. Kluwer Academic Publishers, Dordrecht, 1998.

[6] A. M. Frisch. The substitutional framework for sorted deduction: fundamental results on hybrid reasoning. Artificial Intelligence, 49:161-198, 1991.

[7] A. Galton. Operators vs arguments: The ins and outs of reification. Synthese, 150(3):415-441, 2006.

[8] J. W. Garson. Quantification in modal logic. In D. Gabbay and F. Guenthner, editors, Handbook of Philosophical Logic, Vol.II, pages 249-307. 1984.

[9] J. A. Goguen and J. Meseguer. Order-sorted algebra I: Equational deduction for multiple inheritance, overloading, exceptions and partial operations. Theor. Comput. Sci., 105(2):217-273, 1992. 
[10] J. Y. Halpern and Y. Moses. A guide to completeness and complexity for model logics of knowledge and belief. Artificial Intelligence, 54(3):319-379, April 1992.

[11] K. Kaneiwa. Order-sorted logic programming with predicate hierarchy. Artificial Intelligence, 158(2):155-188, 2004.

[12] K. Kaneiwa and R. Mizoguchi. Ontological knowledge base reasoning with sort-hierarchy and rigidity. In Proceedings of the 9th International Conference on the Principles of Knowledge Representation and Reasoning (KR2004), pages 278-288, 2004.

[13] K. Kaneiwa and R. Mizoguchi. An order-sorted quantified modal logic for meta-ontology. In Proceedings of the International Conference on Automated Reasoning with Analytic Tableaux and Related Methods (TABLEAUX2005), pages 169-184. LNCS 3702, Springer-Verlag, 2005.

[14] K. Kaneiwa and R. Mizoguchi. Distributed reasoning with ontologies and rules in order-sorted logic programming. Journal of Web Semantics, $7(3): 252-270,2009$.

[15] K. Kaneiwa and P.H.P. Nguyen. Decidable order-sorted logic programming for ontologies and rules with argument restructuring. In Proceedings of the 8th International Semantic Web Conference (ISWC 2009), LNCS 5823, pages 328-343. Springer, 2009.

[16] K. Kaneiwa and K. Satoh, On the complexities of consistency checking for restricted UML class diagrams Theoretical Computer Science 411(2), pp. 301-323, 2010.

[17] A. Oberschelp. Untersuchungen zur mehrsortigen Quantorenlogik. Mathematische Annalen 145, pages 297-333, 1962.

[18] A. Oberschelp. Order sorted predicate logic. In Proceedings of Workshop on Sorts and Types in Artificial Intelligence, LNCS 418, pages 8-17. Springer, 1989.

[19] T. Ågotnes. Action and knowledge in alternating-time temporal logic. Synthese, 142(2):375-407, 2006.

[20] M. Schmidt-Schauss. Computational Aspects of an Order-Sorted Logic with Term Declarations. Springer-Verlag, 1989.

[21] A. Sharpanskykh and J. Treur. Relating cognitive process models to behavioural models of agents. In Proceedings of the 2008 IEEE/WIC/ACM International Conference on Intelligent Agent Technology, pages 330-335, 2008. 
[22] G. Smolka. Logic Programming over Polymorphically Order-Sorted Types. PhD thesis, Universitat Kaiserslautern., 1989.

[23] C. Walther. A mechanical solution of Schubert's steamroller by manysorted resolution. Artificial Intelligence, 26(2):217-224, 1985.

[24] C. Walther. A Many-Sorted Calculus Based on Resolution and Paramodulation. Pitman and Kaufman Publishers, 1987.

[25] C. Walther. Many-sorted unification. Journal of the Association for Computing Machinery, 35:1, 1988.

[26] T. Weibel. An order-sorted resolution in theory and practice. Theoretical Computer Science, 185(2):393-410, 1997.

[27] C. Weidenbach. First-order tableaux with sorts. Journal of the Interest Group in Pure and Applied Logics, 3(6):887-906, 1995.

[28] C. Weidenbach. Combining Superposition, Sorts and Splitting, volume 1, chapter 27, pages 1965-2013. Elsevier, Amsterdam, the Netherlands, January 2001.

[29] C. Welty and W. Andersen. Towards ontoclean 2.0: A framework for rigidity. Applied Ontology, 1(1):107-116, 2005.

[30] C. Welty and N. Guarino. Supporting ontological analysis of taxonomic relationships. Data and Knowledge Engineering, 39(1):51-74, 2001. 